\title{
Harmonic morphisms with fibers of dimension one
}

\author{
ROBERT L. BRYANT ${ }^{1}$
}

\begin{abstract}
The harmonic morphisms $\phi: M^{n+1} \rightarrow N^{n}$ are studied using the methods of the moving frame and exterior differential systems and three main results are achieved.

The first result is a local structure theorem for such maps in the case that $\phi$ is a submersion, in particular, a normal form is found for all such $\phi$ once the metric on the target manifold $N$ is specified. The second result is a finiteness theorem, which says, in a certain sense, that, when $n \geq 3$, the set of harmonic morphisms with a given Riemannian domain $\left(M^{n+1}, g\right)$ is a finite dimensional space. The third result is the explicit classification when $n \geq 3$ of all local and global harmonic morphisms with domain $\left(M^{n+1}, g\right)$, a space of constant curvature.
\end{abstract}

\section{Introduction.}

A smooth map $\phi: M \rightarrow N$ between Riemannian manifolds is said to be a harmonic morphism if, for any harmonic function $f$ on any open set $V \subset N$, the pullback $f \circ \phi$ is a harmonic function on $\phi^{-1}(V) \subset M$.

By a simple argument (see $\S 1$ ), any non-constant harmonic morphism $\phi$ : $M \rightarrow N$ between connected Riemannian manifolds must be a submersion away from a set of measure zero in $M$. Thus, a necessary condition for the existence of a non-constant harmonic map $\phi: M \rightarrow N$ is that $\operatorname{dim} M \geq$ $\operatorname{dim} N$.

When the dimension of $N$ is 1 , so that $N$ can be regarded, at least locally, as $\mathbb{R}$ with its standard metric, a map $\phi: M \rightarrow N$ is a harmonic morphism if and only if it is a harmonic function in the usual sense. Thus, at least locally, there are many harmonic morphisms from $M$ to $N$.

\footnotetext{
${ }^{1}$ This research was begun during a visit to IMPA in Rio de Janeiro in July 1996 and was inspired by questions raised during the International Conference on Differential Geometry held at IMPA during that month. The article was written during a visit to the Institute for Advanced Study in Princeton. The author would like to thank IMPA and the IAS for their hospitality and also to acknowledge support from the National Science Foundation through grant DMS-9505125.
} 
However, when the dimension of $N$ is greater than 1 , the condition of being a harmonic morphism turns out to be much more restrictive, being essentially equivalent to an overdetermined system of PDE for the map $\phi$. Thus, for generic Riemannian metrics on $M$ and $N$, one does not expect there to be any harmonic morphisms, even locally. Moreover, in the case that there do exist harmonic morphisms $\phi: M \rightarrow N$ for given $M$ and $N$, one expects the analysis of the overdetermined system that describes them to involve integrability conditions and other features of overdetermined systems.

When both $M$ and $N$ have dimension 2, a harmonic morphism is simply a branched conformal mapping between Riemann surfaces and these are studied by classical methods of complex analysis and Riemann surface theory.

When both manifolds have the same dimension $n>2$, a non-constant harmonic morphism is a local homothety, i.e., up to a constant scale factor, $\phi$ is a local isometry.

Thus, the interesting cases are when $\operatorname{dim} M>\operatorname{dim} N \geq 2$. This article concerns the case when $\operatorname{dim} M=\operatorname{dim} N+1$, i.e., when the dimension of the generic fiber of $\phi$ is 1 . It contains three main results.

The first, Theorem 1, is a local structure theorem for harmonic morphisms whose fibers are curves. This result describes the possible Riemannian metrics $g$ that can be defined on the domain $M$ of a smooth mapping $\phi: M \rightarrow N$ where $N$ is a smooth manifold endowed with a fixed Riemannian metric $h$ so that $\phi:(M, g) \rightarrow(N, h)$ will be a harmonic morphism.

The second result, Theorem 2 , is a general finiteness theorem for harmonic morphisms of corank one with a given Riemannian domain $\left(M^{n+1}, g\right)$ where $n \geq 3$. This result shows that the set of such harmonic morphisms is, in a certain sense, finite dimensional. This result is in marked contrast to the case $n=2$, which has already been analyzed by Baird and Wood with the result that the locally defined harmonic morphisms with a given Riemannian domain $\left(M^{3}, g\right)$ of constant sectional curvature depend on arbitrary functions (in the sense of exterior differential systems).

The third result, Theorem 3, is a classification of the harmonic morphisms of corank one whose domain $\left(M^{n+1}, g\right)$ is a simply-connected, complete Riemannian manifold of constant curvature and dimension $n+1 \geq 4$. It will be shown that there are exactly two types of such harmonic morphisms.

The first type can be thought of as a sort of metric quotient and is described as follows: Let $X$ be a Killing vector field on $M$ with zero locus $Z \subset M$ and suppose that the space $N$ of integral curves of $X$ in $M \backslash Z$ can 
be given the structure of a smooth $n$-manifold in such a way that the quotient map $\phi: M \backslash Z \rightarrow N$ is a smooth submersion. Then there exists a metric $h$ on $N$, unique up to a constant scale factor, so that $\phi:(M \backslash Z, g) \rightarrow(N, h)$ is a harmonic morphism. (Sometimes this map can be extended across $Z$ as well after suitably extending $N$, see $\S 3.3$.) The second type is described as follows: Let $N \subset M$ be a totally umbilic hypersurface, endowed with a constant multiple of the induced metric, denoted $h$. Let $P \subset M$ be the focal set of $N$, which consists of at most two points. There is a canonical retraction $\phi: M \backslash P \rightarrow N$ that retracts $M \backslash P$ back to $N$ along the geodesics normal to $N$. Then $\phi$ is a harmonic morphism. The examples of this kind had already appeared in the work of Gudmundsson [Gu1].

The methods used are those of exterior differential systems and the moving frame, both of which are well-adapted to the study of overdetermined systems of PDE.

Acknowledgments. It is a pleasure to thank John Wood, whose questions inspired this article and whose comments and and guide to the literature on harmonic morphisms were invaluable.

\section{Harmonic morphisms via moving frames.}

This section is a self-contained treatment by moving frame calculations of the the basic structure theory of harmonic morphisms. It is intended to be readable by those familiar with either moving frame calculations or the fundamentals of harmonic morphisms.

Its main purpose is to fix notation and to serve as a reference for the proofs in the later sections, which employ the moving frame. Such a reference is probably needed, as it appears that most of the current workers on harmonic morphisms do not use moving frames and so the translation of known results into this language may be helpful. For more background on the method of the moving frame, see [Sp]. Most of the results about harmonic maps and morphisms to be derived in this section can be found in the standard references on the subject, such as [EL1], [EL2], or [W2].

\subsection{Moving frame computations for harmonic morphisms.}

Let $M$ and $N$ be Riemannian manifolds of dimensions $m$ and $n$, respectively. For simplicity, I assume that both $M$ and $N$ are connected throughout this article. The summation convention will be used extensively, with the 
understood ranges

$$
\begin{aligned}
& 1 \leq a, b, c \leq m, \\
& 1 \leq i, j, k \leq n .
\end{aligned}
$$

1.1.1. Coframings, connection forms, and structure equations. Let $g$ be the metric on $M$ and $h$ be the metric on $N$. Let $U \subset M$ and $V \subset N$ be open sets with trivial tangent bundles. Then there exist smooth coframings $\omega=\left(\omega_{1}, \ldots, \omega_{m}\right)$ and $\eta=\left(\eta_{1}, \ldots, \eta_{n}\right)$ on $U$ and $V$ respectively, so that

$$
\begin{aligned}
& g_{\mid U}=\omega_{1}^{2}+\cdots+\omega_{m}^{2}=\omega_{a}{ }^{2} \\
& h_{\mid V}=\eta_{1}^{2}+\cdots+\eta_{n}^{2}=\eta_{i}{ }^{2} .
\end{aligned}
$$

Corresponding to the chosen coframings on the respective open sets, there exist unique 1-forms $\omega_{a b}=-\omega_{b a}$ and $\eta_{i j}=-\eta_{j i}$ that represent the LeviCivita connections of the respective metrics and that are characterized by the structure equations

$$
d \omega_{a}=-\omega_{a b} \wedge \omega_{b}, \quad d \eta_{i}=-\eta_{i j} \wedge \eta_{j} .
$$

1.1.2. Mappings and pullbacks. Now suppose that $\phi: M \rightarrow N$ is a smooth map and that $U$ and $V$ have been chosen so that $U \subset f^{-1}(V)$. Then there exist unique functions $f_{i a}$ on $U$ so that

$$
\phi^{*}\left(\eta_{i}\right)=f_{i a} \omega_{a} .
$$

Because the chosen coframings are orthonormal, the energy density of the map $\phi$ on $U$ is given by

$$
\begin{aligned}
E(\phi)_{\mid U} & =f_{i a} f_{i a}\left|\omega_{1} \wedge \ldots \wedge \omega_{n}\right| \\
& =\left\|\phi^{\prime}\right\|^{2}|U| \omega_{1} \wedge \ldots \wedge \omega_{n} \mid .
\end{aligned}
$$

This density is globally defined, independent of the local choice of $\omega$ or $\eta$. When $M$ is compact, integration of this density yields a functional called. the energy $\mathcal{E}: C^{\infty}(M, N) \rightarrow \mathbb{R}$, namely

$$
\mathcal{E}(\phi)=\frac{1}{2} \int_{M} E(\phi)
$$

Adopt the convention that, for any differential form $\psi$ on $V$, its $\phi$ pullback $\phi^{*}(\psi)$ on $U$ is denoted by an overbar, i.e., $\bar{\psi}=\phi^{*}(\psi)$. Since 
the map $\phi$ will be fixed in this discussion, this should cause no confusion. Thus, (3) becomes

$$
\overline{\eta_{i}}=f_{i a} \omega_{a}
$$

(The reader of other sources on moving frame calculations should be aware that many authors simply drop the pullback notation entirely, writing (3) in the even simpler form $\eta_{i}=f_{i a} \omega_{a}$. This has caused considerable confusion in some cases, a confusion I hope to avoid.)

Taking the exterior derivative of (3) and using the structure equations (2) yields

$$
\left(d f_{i a}-f_{i b} \omega_{b a}+f_{j a} \overline{\eta_{i j}}\right) \wedge \omega_{a}=0 .
$$

By Cartan's Lemma, there exist unique functions $f_{i a b}=f_{i b a}$ on $U$ so that

$$
d f_{i a}=f_{i b} \omega_{b a}-f_{j a} \overline{\eta_{i j}}+f_{i a b} \omega_{b} .
$$

The tension field of $\phi$ on $U$ is the tensor field

$$
\tau(\phi)=f_{i a a} \quad \mathbf{e}_{i} \circ \phi
$$

(where $\left(\mathbf{e}_{1}, \ldots, \mathbf{e}_{n}\right)$ is the dual orthonormal frame field in $V$ to $\eta$ ) and is a section of the $\phi$-pullback to $U$ of the tangent bundle of $N$. This tensor does not depend on the choice of $\omega$ or $\eta$ and so is globally defined on $M$.

The map $\phi$ is said to be harmonic if it satisfies the Euler-Lagrange equations for the energy functional $\mathcal{E}$. A calculation (see [EL2,(2.30)]) shows that $\phi$ is harmonic if and only if its tension field vanishes. A function $f$ : $N \rightarrow \mathbb{R}$ is said to be harmonic if it is harmonic as a map to $\mathbb{R}$ endowed with its standard metric.

1.1.3. Harmonic morphisms. It is not generally true that the composition of harmonic maps is harmonic, so there is no useful category whose objects are the Riemannian manifolds and whose morphisms are harmonic maps. Nevertheless, some harmonic maps turn out to have useful composition properties and these are designated as harmonic morphisms. In this subsection, this notion will be explained and the equations for it derived.

Given a smooth function $v$ on $V$, there exist functions $v_{i}$ so that $d v=$ $v_{i} \eta_{i}$. Differentiating this relation gives $0=\left(d v_{i}-v_{k} \eta_{k i}\right) \wedge \eta_{i}$. By Cartan's Lemma, there exist functions $v_{i j}=v_{j i}$ so that $d v_{i}=v_{k} \eta_{k i}+v_{i j} \eta_{j}$. The trace $\Delta v=-v_{i i}$ is the local expression with respect to the coframing $\eta$ for the globally defined $h$-Laplacian of $v$, which is also the tension field of $v$ 
regarded as a mapping $v: V \rightarrow \mathbb{R}$. Thus, $v$ is harmonic in $V$ if and only if $\Delta v$ vanishes identically in $V$.

Locally, there are many harmonic functions. It is known [EL1] that, for any $q \in V$, and any collection of real numbers $r_{i}$ and $r_{i j}=r_{j i}$ with $r_{i i}=0$, there is an open $q$-neighborhood $V^{\prime} \subset V$ and a harmonic function $v$ on $V^{\prime}$ so that $v_{i}(q)=r_{i}$ and $v_{i j}(q)=r_{i j}$. This local 'flexibility' will be important below.

Roughly speaking, a map $\phi: M \rightarrow N$ will be a harmonic 'morphism' if $\phi$-pullback carries harmonic functions on $N$ to harmonic functions on $M$. The precise definition is as follows:

Definition 1. A map $\phi: M \rightarrow N$ is a harmonic morphism if, for any harmonic function $v$ on an open set $V \subset N$, the function $\bar{v}=\phi^{*}(v)$ is harmonic on $\phi^{-1}(V)$ in $M$.

It is easy to derive the partial differential equations that characterize harmonic morphisms in terms of local coframings. Suppose that $U$ and $V$ are endowed with orthonormal coframings as above. Suppose, further, that $v$ is any harmonic function on $V$ and keep the notation as in the previous paragraph. Set $u=\phi^{*}(v)=\bar{v}$. Pulling back the relation $d v=v_{i} \eta_{i}$ then yields

$$
d u=f_{i a} \overline{v_{i}} \omega_{a}
$$

so $u_{a}=f_{i a} \overline{v_{i}}$. Differentiating this relation and comparing the result with the pullback of the relation $d v_{i}=v_{k} \eta_{k i}+v_{i j} \eta_{j}$ then yields

$$
u_{a b}=f_{i a} f_{j b} \overline{v_{i j}}+f_{i a b} \overline{v_{i}} .
$$

In particular,

$$
\Delta u=-f_{i a} f_{j a} \overline{v_{i j}}-f_{i a a} \overline{v_{i}} .
$$

Now, because of the above-mentioned flexibility in choosing the 2-jet of a local harmonic function, it follows that a necessary and sufficient condition for $\phi: U \rightarrow V$ to be a harmonic morphism is that there exist a function $R$ on $U$ so that the right hand side of $(6)$ is of the form $-R \overline{v_{i i}}$. In other words,

(7) $f_{i a a}=0$ and $\quad f_{i a} f_{j a}=R \delta_{i j}$ for some function $R \geq 0$ on $U$.

The first condition in (7) is just that $\phi$ be a harmonic map. The second condition is known in the literature as horizontal weak conformality, since 
it says that, for all $p \in M$, the differential $\phi^{\prime}(p): T_{p} M \rightarrow T_{\phi(p)} N$ can be geometrically described as orthogonal projection from the kernel of $\phi^{\prime}(p)$ to its orthogonal complement followed by an isometry plus a conformal scaling. This characterization of harmonic morphisms is due, independently, to Fuglede [Fu] and Ishihara [Is] and can be found in [EL1,(4.12)].

Note that the conformal factor $R$ defined in (7) satisfies $n R=\left\|\phi^{\prime}\right\|^{2}$ and hence vanishes only at the points where $\phi^{\prime}$ vanishes. According to [EL2,(2.32)], if $R$ vanishes on an open set, then it vanishes identically on $M$ (since $M$ is connected). (This sort of 'unique continuation' principle holds more generally for all harmonic maps, see [EL1,(3.16-18)].) Set aside this trivial case, in which $\phi$ is constant, and assume from now on that $\phi$ is non-constant. Thus, the set where $R>0$ is a dense open subset $M^{*}$ in $M$.

By (7), the harmonic morphism $\phi: M^{*} \rightarrow N$ is a submersion, so the dimension of $M$ must satisfy $m=n+p \geq n$. The number $p$ will be called the fiber rank or corank of $\phi$.

1.1.4. Horizontal conformality. Now, the conditions (7) form an overdetermined system of PDE for the map $\phi$. The first condition, harmonicity, is second order and the second condition, horizontal (weak) conformality, is first order. The first step in studying this system is to examine the consequences of the first order conditions.

Expand the summation convention to let lower case Greek indices run over the range $n<\alpha, \beta, \gamma \leq n+p$. (When $p=0$, this range is empty, so the formulae below have to be modified slightly in various obvious ways, a task left to the reader.)

Assume that the open set $V \subset N$ has a trivial tangent bundle and that the rank $p$ subbundle ker $\phi^{\prime} \subset T U$ is trivial on the open set $U \subset \phi^{-1}(V) \cap M^{*}$. Let $r>0$ be the function on $U$ satisfying $n r^{2}=\left\|\phi^{\prime}\right\|^{2}$. Then the 1-forms $\omega_{i}=r^{-1} \overline{\eta_{i}}=r^{-1} \phi^{*}\left(\eta_{i}\right)$ are $g$-orthonormal on $U$ and so, because of the triviality of the bundle ker $\phi^{\prime}$, they can be completed to a $g$-orthonormal coframing by choosing $p$ additional 1 -forms $\omega_{n+1}, \ldots, \omega_{n+p}$ so that

$$
g_{\mid U}=\omega_{1}^{2}+\cdots+\omega_{n+p}^{2}=\omega_{i}^{2}+\omega_{\alpha}^{2}
$$

I will say that such a pair of coframings $\omega$ on $U$ and $\eta$ on $V$ is $\phi$-adapted. For a $\phi$-adapted pair of coframings, (3) simplifies to

$$
f_{i a}=r \delta_{i a}
$$


Set $r^{-1} d r=r_{a} \omega_{a}=r_{i} \omega_{i}+r_{\alpha} \omega_{\alpha}$. Differentiating $\overline{\eta_{i}}=r \omega_{i}$ yields

$$
\begin{aligned}
d\left(\overline{\eta_{i}}\right) & =d r \wedge \omega_{i}+r d \omega_{i} \\
r^{-1}\left(-\overline{\eta_{i j}} \wedge \overline{\eta_{j}}\right) & =r^{-1} d r \wedge \omega_{i}-\omega_{i j} \wedge \omega_{j}-\omega_{i \alpha} \wedge \omega_{\alpha} \\
-\overline{\eta_{i j}} \wedge \omega_{j} & =\left(r_{i} \omega_{i}+r_{\alpha} \omega_{\alpha}\right) \wedge \omega_{i}-\omega_{i j} \wedge \omega_{j}-\omega_{i \alpha} \wedge \omega_{\alpha} \\
0 & =\left[\left(r_{k} \omega_{k}+r_{\alpha} \omega_{\alpha}\right) \delta_{i j}+\overline{\eta_{i j}}-\omega_{i j}\right] \wedge \omega_{j}-\omega_{i \alpha} \wedge \omega_{\alpha} .
\end{aligned}
$$

Since $\omega_{i \alpha} \wedge \omega_{\alpha} \equiv 0 \bmod \omega_{1}, \ldots, \omega_{n}$, Cartan's Lemma implies that there exist functions $A_{i j \alpha}$ and $H_{i \alpha \beta}=H_{i \beta \alpha}$ on $U$ so that

$$
\omega_{i \alpha}=A_{i j \alpha} \omega_{j}+H_{i \alpha \beta} \omega_{\beta} \text {. }
$$

The functions $A_{i j \alpha}$ and $H_{i \alpha \beta}$ are the local components of globally defined tensor fields: The expression

$$
\mathbf{H}=H_{i \alpha \beta} \omega_{\alpha} \circ \omega_{\beta} \otimes \mathbf{e}_{i}
$$

is independent of choice of frame field and has the defining property that it restricts to each fiber of $\phi$ to be the second fundamental form of that fiber in $M^{*}$. The $i j$-skewsymmetrization of the expression

$$
\mathbf{A}=A_{i j \alpha} \omega_{i} \otimes \omega_{j} \otimes \mathbf{e}_{\alpha}
$$

is the integrability tensor of the $n$-plane field $H_{\phi}=\left(\operatorname{ker} \phi^{\prime}\right)^{\perp}$. (The symmetric part will be examined below.)

Substituting (10) into (9) yields the relation

$$
0=\left[\left(r_{k} \omega_{k}+r_{\alpha} \omega_{\alpha}\right) \delta_{i j}+\overline{\eta_{i j}}-\omega_{i j}+A_{i j \alpha} \omega_{\alpha}\right] \wedge \omega_{j}
$$

and applying Cartan's Lemma to this yields that there exist functions $s_{i j k}=$ $s_{i k j}$ so that

$$
\left(r_{k} \omega_{k}+r_{\alpha} \omega_{\alpha}\right) \delta_{i j}+\overline{\eta_{i j}}-\omega_{i j}+A_{i j \alpha} \omega_{\alpha}=s_{i j k} \omega_{k} .
$$

Symmetrizing the $\omega_{\alpha}$-components on both sides of this expression yields

$$
2 \delta_{i j} r_{\alpha}+A_{i j \alpha}+A_{j i \alpha}=0,
$$

implying that there are functions $a_{i j \alpha}=-a_{j i \alpha}$ so that $A_{i j \alpha}=-\delta_{i j} r_{\alpha}+a_{i j \alpha}$. This gives a sharpened version of (10), which now takes the form

$$
\omega_{i \alpha}=-r_{\alpha} \omega_{i}+a_{i j \alpha} \omega_{j}+H_{i \alpha \beta} \omega_{\beta}, \quad \text { with } a_{i j \alpha}=-a_{j i \alpha} .
$$

Note that the skewsymmetric tensor $\mathbf{A}^{\prime}=a_{i j \alpha} \omega_{i} \wedge \omega_{j} \otimes \mathbf{e}_{\alpha}$ is the integrability tensor for the $n$-plane field $H_{\phi}$.

Substituting $\left(10^{\prime}\right)$ back into (11) allows one to solve for the $s_{i j k}$, finally yielding the relation

$$
\overline{\eta_{i j}}=\omega_{i j}+a_{i j \alpha} \omega_{\alpha}+r_{j} \omega_{i}-r_{i} \omega_{j}
$$


1.1.5. Harmonicity. With this information derived from horizontal conformality, the quantities $f_{i a b}$ can now be computed via the defining formula (4):

$$
\begin{aligned}
f_{i a b} \omega_{b} & =d f_{i a}-f_{i b} \omega_{b a}+f_{j a} \overline{\eta_{i j}}=d\left(r \delta_{i a}\right)-r \delta_{i b} \omega_{b a}+r \delta_{j a} \overline{\eta_{i j}} \\
& =r\left(\delta_{i a}\left(r_{b} \omega_{b}\right)-\omega_{i a}+\delta_{j a} \overline{\eta_{i j}}\right)
\end{aligned}
$$

In particular, taking $a=\alpha>n$ and using $\left(10^{\prime}\right)$,

$$
f_{i \alpha b} \omega_{b}=-r \omega_{i \alpha}=r r_{\alpha} \omega_{i}-r a_{i j \alpha} \omega_{j}-r H_{i \alpha \beta} \omega_{\beta},
$$

so that $f_{i \alpha \beta}=-r H_{i \alpha \beta}$. Next, taking $a=j \leq n$, and using (12),

$$
\begin{aligned}
f_{i j b} \omega_{b} & =r\left(\delta_{i j}\left(r_{b} \omega_{b}\right)-\omega_{i j}+\overline{\eta_{i j}}\right) \\
& =r\left(\delta_{i j}\left(r_{b} \omega_{b}\right) a_{i j \alpha} \omega_{\alpha}+r_{j} \omega_{i}-r_{i} \omega_{j}\right) .
\end{aligned}
$$

so that, in particular, $f_{i j k}=r\left(\delta_{i j} r_{k}+\delta_{i k} r_{j}-\delta_{k j} r_{i}\right)$.

The components of the tension field $\tau(\phi)$ can now expressed as

$$
f_{i a a}=f_{i j j}+f_{i \alpha \alpha}=(2-n) r r_{i}-r H_{i \alpha \alpha}=-r\left((n-2) r_{i}+H_{i \alpha \alpha}\right) .
$$

Thus, a horizontally conformal submersion $\phi$ is a harmonic morphism if and only if it satisfies $(n-2) r_{i}+H_{i \alpha \alpha}=0$.

1.1.6. Elementary consequences. Note that (13) yields the well-known result that, when $n=2$, a horizontally conformal map is a harmonic morphism if and only if the fibers are minimal submanifolds, i.e., the trace of the second fundamental form of each fiber vanishes [EL2,(2.34)]. Consequently, when $n=2$, only the conformal structure on $N$ is involved in determining whether or not the map $\phi$ is a harmonic morphism, not the full metric on $N$.

Another consequence involves the case $p=0$, for, in this case, (13) has no terms $H_{i \alpha \alpha}$. Thus, in order for $\phi$ to be a non-constant harmonic morphism between two manifolds of equal dimension $n \neq 2$, the terms $r_{i}$ must all vanish, so that the conformal factor $r$ is constant. Thus, when $n \neq 2$, any non-constant harmonic morphism $\phi: M^{n} \rightarrow N^{n}$ must be a homothety, i.e., satisfy $\phi^{*} h=r^{2} g$ for some constant $r>0$.

On the other hand, when $p=0$ and $n=2$, equation (13) shows that $f_{i a a}=0$ is an identity, so that the only condition for a map between Riemannian surfaces to be a harmonic morphism is that the map be a branched conformal map. This is also well-known [EL1]. 
Finally note that, when $n=1$, the condition of horizontal conformality is automatic, so that a harmonic morphism is the same thing as a harmonic map.

Remark. One geometric interpretation of (13) is that, for a harmonic morphism $\phi: M^{*} \rightarrow N$, parallel translation conserves volume in the fibers of $\phi$ when it is measured with respect to the $p$-form $r^{2-n} \omega_{n+1} \wedge \ldots \wedge \omega_{n+p}$. This observation will be useful in $\S 2$.

\subsection{Moving frame computations for conformal foliations.}

The goal of this subsection is to derive necessary and sufficient conditions on the invariants of a foliation of a Riemannian manifold $M$ in order that its leaves be locally the fibers of some submersive harmonic morphism.

It is useful to introduce a bit of terminology about foliations. A foliation $\mathcal{F}$ of codimension $n$ on a manifold $M^{n+p}$ is said to be amenable if the leaf space $M / \mathcal{F}$ can be given the structure of a smooth $n$-manifold in such a way that the leaf projection $M \rightarrow M / \mathcal{F}$ becomes a smooth submersion. When a smooth structure on $M / \mathcal{F}$ does exist with this property, it is unique. Every foliation is locally amenable in the sense that, for any smooth foliation $\mathcal{F}$ on $M$, each point of $M$ has an open neighborhood $U$ on which $\mathcal{F}$ is amenable. Such an open set $U$ will be said to be $\mathcal{F}$-amenable.

Now, suppose that $\mathcal{F}$ be a foliation of codimension $n$ on a manifold $M^{n+p}$ endowed with a Riemannian metric $g$. Using the metric $g$, the tangent bundle of $M$ can be split as a $g$-orthogonal direct sum

$$
T M=T \mathcal{F} \oplus N \mathcal{F}
$$

where $T \mathcal{F}$ and $N \mathcal{F}$ are the tangent and normal bundles of the foliation $\mathcal{F}$, respectively. Correspondingly, the metric $g$ can be split into two terms

$$
g=g^{\prime}+g^{\prime \prime}
$$

where the quadratic form $g^{\prime}$ has $T \mathcal{F}$ as its null space and $g^{\prime \prime}$ has $N \mathcal{F}$ as its null space.

1.2.1. Conformal foliations. Let $U \subset M$ be an open set on which $T \mathcal{F}$ and $N \mathcal{F}$ are both trivial. Then there exists a $g$-orthonormal coframing $\omega=\left(\omega_{1}, \ldots, \omega_{n+p}\right)$ on $U$ with dual frame field $\mathbf{e}=\left(\mathbf{e}_{1}, \ldots, \mathbf{e}_{n+p}\right)$ so that the equations $\omega_{1}=\omega_{2}=\cdots=\omega_{n}=0$ define $T \mathcal{F}$ while the equations $\omega_{n+1}=\omega_{n+2}=\cdots=\omega_{n+p}=0$ define $N \mathcal{F}$. In particular, the formula

$$
g^{\prime}=\omega_{1}^{2}+\cdots+\omega_{n}^{2}
$$


holds on $U$. The equations $d \omega_{a}=-\omega_{a b} \wedge \omega_{b}$ imply (keeping the previously established index ranges for the summation convention)

$$
d \omega_{i}=-\omega_{i a} \wedge \omega_{a} \equiv-\omega_{i \alpha} \wedge \omega_{\alpha} \bmod \omega_{1}, \ldots, \omega_{n} .
$$

Since the $\omega_{i}$ are the annihilators of a foliation, $d \omega_{i} \equiv 0 \bmod \omega_{1}, \ldots, \omega_{n}$. Thus, $\omega_{i \alpha} \wedge \omega_{\alpha} \equiv 0 \bmod \omega_{1}, \ldots, \omega_{n}$, so by Cartan's Lemma there exist functions $A_{i j \alpha}$ and $H_{i \alpha \beta}=H_{i \beta \alpha}$ so that

$$
\omega_{i \alpha}=A_{i j \alpha} \omega_{j}+H_{i \alpha \beta} \omega_{\beta} .
$$

Now, the discussion from $\S 1.1$ shows that a necessary condition that the leaves of $\mathcal{F}$ in $U$ be the fibers of a submersive harmonic morphism with domain $U$ is that there exist functions $r_{\alpha}$ on $U$ so that

$$
A_{i j \alpha}+A_{j i \alpha}=-2 r_{\alpha} \delta_{i j} .
$$

Geometrically, condition (2) can be interpreted as the condition that the Lie derivative of $g^{\prime}$ with respect to any vector field $X=x_{\alpha} \mathbf{e}_{\alpha}$ (tangent to the leaves of $\mathcal{F}$ ) should be a multiple of $g^{\prime}$. Indeed, calculation yields

$$
\begin{aligned}
\mathcal{L}_{X}\left(g^{\prime}\right) & \left.\left.=2\left(\mathcal{L}_{X} \omega_{i}\right) \circ \omega_{i}=2(X\lrcorner d \omega_{i}\right) \circ \omega_{i}=-2(X\lrcorner\left(\omega_{i a} \wedge \omega_{a}\right)\right) \circ \omega_{i} \\
& =-2\left(\omega_{i a}(X) \omega_{a}-\omega_{a}(X) \omega_{i a}\right) \circ \omega_{i} \\
& =-2\left(\omega_{i \alpha}(X) \omega_{\alpha}-x_{\alpha} \omega_{i \alpha}\right) \circ \omega_{i} \\
& =-2\left(H_{i \alpha \beta} x_{\beta} \omega_{\alpha}-x_{\alpha}\left(A_{i j \alpha} \omega_{j}+H_{i \alpha \beta} \omega_{\beta}\right)\right) \circ \omega_{i} \\
& =x_{\alpha}\left(A_{i j \alpha}+A_{j i \alpha}\right) \omega_{i} \circ \omega_{j}
\end{aligned}
$$

and this final expression is a multiple of $g^{\prime}$ for any choice of the functions $x_{\alpha}$ if and only if (2) is satisfied for some functions $r_{\alpha}$ on $U$.

This local condition can be expressed globally on $M$. If (2) is satisfied for some functions $r_{\alpha}$, then the 1-form $\rho^{\prime \prime}=r_{\alpha} \omega_{\alpha}$ satisfies

$$
\mathcal{L}_{X}\left(g^{\prime}\right)=-2 \rho^{\prime \prime}(X) g^{\prime}
$$

for all vector fields $X$ tangent to the leaves of $\mathcal{F}$. Now, the 1 -form $\rho^{\prime \prime}$ is independent of the choice of $\omega$. Thus, the global version of (2) is that there should exist a 1-form $\rho^{\prime \prime}$ on $M$ (necessarily unique) that vanishes on $N \mathcal{F}$ and so that $\left(2^{\prime}\right)$ is satisfied for all vector fields $X$ tangent to the leaves of $\mathcal{F}$.

Geometrically, condition $\left(2^{\prime}\right)$ is satisfied if and only if, up to a conformal factor, the quadratic form $g^{\prime}$ can be pushed down onto the local leaf space $U / \mathcal{F}$ for any $\mathcal{F}$-amenable $U \subset M$. A foliation $\mathcal{F}$ satisfying $\left(2^{\prime}\right)$ with respect to a given metric $g$ is sometimes called a conformal foliation and this constitutes a first necessary condition in order for the leaves of $\mathcal{F}$ to be the fibers of a harmonic morphism. 
1.2.2. Local sufficient conditions. To go further, the analysis must be divided into the cases $n=2$ and $n \neq 2$.

First, consider the case $n=2$. In $\S 1.1$, it was shown that the non-singular fibers of a harmonic morphism $\phi: M^{2+p} \rightarrow N^{2}$ are minimal submanifolds of codimension 2, so it is necessary that the leaves of a codimension 2 foliation $\mathcal{F}$ on $M$ be minimal in order for them to be the fibers of a (local) submersive harmonic morphism. Of course, $\mathcal{F}$ must also be a conformal foliation.

Conversely, as has long been known [W1] (for example), these two conditions are locally sufficient.

Proposition 1. Suppose that $\mathcal{F}$ is a codimension 2 conformal foliation of $M^{2+p}$ with the property that its leaves are minimal. Then, for every open $U \subset M$ that is $\mathcal{F}$-amenable, there is a conformal structure on the 2dimensional manifold $U / \mathcal{F}$ so that the leaf projection $\phi: U \rightarrow U / \mathcal{F}$ is a harmonic morphism.

Proof. This follows immediately from the discussion so far. If $U \subset M$ is an $\mathcal{F}$-amenable open set, then $\left(2^{\prime}\right)$ implies that $U / \mathcal{F}$ carries a unique conformal structure so that the leaf projection $\phi$ is horizontally conformal. From the discussion in $\S 1.1$ after equation (13), the only other condition for $\phi$ to be a harmonic morphism is that its fibers be minimal.

Second, consider the case $n \neq 2$ and restrict to $U$ with a coframing as in $\S 1.2 .1$. Define functions $r_{i}$ on $U$ by $(n-2) r_{i}=-H_{i \alpha \alpha}$. The vector field $\mathbf{R}=r_{i} \mathbf{e}_{i}$ is, up to a constant scale factor, the mean curvature normal vector field for the leaves of $\mathcal{F}$. Let $\rho^{\prime}=r_{i} \omega_{i}$. This 1 -form is locally defined with respect to a coframing, but, since it is the $g$-dual of the mean curvature vector field for the leaves of $\mathcal{F}$, it is, in fact, globally defined on $M$. According to (13) of $\S 1.1$, for any harmonic morphism $\phi: M \rightarrow N$ whose fibers are the leaves of $\mathcal{F}$, the 1 -form

$$
\rho=r_{a} \omega_{a}=r_{i} \omega_{i}+r_{\alpha} \omega_{\alpha}=\rho^{\prime}+\rho^{\prime \prime}=\frac{-1}{(n-2)} H_{i \alpha \alpha} \omega_{i}+r_{\alpha} \omega_{\alpha}
$$

must satisfy $\rho=d(\log r)$ where $r^{2} g^{\prime}=\phi^{*} h$ and where $h$ is the metric on the target manifold $N$. Thus, a necessary condition for the leaves of $\mathcal{F}$ to be the fibers of a harmonic morphism is that

$$
d \rho=0 .
$$

The interesting result is the converse (which appears to be new): 
Proposition 2. If a foliation $\mathcal{F}$ of codimension $n \neq 2$ on a Riemannian manifold $(M, g)$ is conformal and satisfies (5), then for any 1-connected, $\mathcal{F}$-amenable open set $U \subset M$, there is a metric $h$ on $U / \mathcal{F}$, unique up to a constant scale factor, for which the leaf projection $\phi: U \rightarrow U / \mathcal{F}$ is a harmonic morphism $\phi:(U, g) \rightarrow(U / \mathcal{F}, h)$.

Proof. Let $U \subset M$ be 1-connected and $\mathcal{F}$-amenable and let $\phi: U \rightarrow U / \mathcal{F}$ be the leaf projection. Since $\mathcal{F}$ is a conformal foliation, $\rho^{\prime \prime}$ can be defined so that $\left(2^{\prime}\right)$ holds while $\rho^{\prime \prime}$ vanishes on $N \mathcal{F}$. Moreover, let $\rho^{\prime}$ be defined as above to be the (suitably scaled) dual to the mean curvature vector field of $\mathcal{F}$. Finally, use (4) to define the 1-form $\rho$. The assumption that (5) holds is just that $d \rho=0$.

By the simple connectivity of $U$, there exists a smooth positive function $r$ on $U$ so that $\rho=d(\log r)$. Since $U$ is connected, $r$ is unique up to a multiplicative constant.

Let $g^{\prime}$ be the leaf normal part of the metric $g$, as defined above. The formula $\left(2^{\prime}\right)$ can be written as

$$
\mathcal{L}_{X}\left(g^{\prime}\right)=-2 \rho(X) g^{\prime}=-2 \mathcal{L}_{X}(\log r) g^{\prime}
$$

for all vector fields $X$ tangent to the leaves of $\mathcal{F}$. It follows that $\mathcal{L}_{X}\left(r^{2} g^{\prime}\right)=0$ for all such vector fields $X$. In particular, there must exist a metric $h$ on $U / \mathcal{F}$ so that $r^{2} g^{\prime}=\phi^{*}(h)$.

The leaf projection $\phi:(U, g) \rightarrow(U / \mathcal{F}, h)$ is horizontally conformal by construction and equation (13) of $\S 1.1$ now shows that $\phi$ is harmonic. Thus, $\phi$ is a harmonic morphism.

The uniqueness of $h$ with this property is immediate.

By Propositions 1 and 2, the foliation $\mathcal{F}$ alone carries enough information to construct any harmonic morphism whose fibers are the leaves of $\mathcal{F}$, locally and essentially uniquely, up to a constant scale factor on the range. This observation will be important in the remainder of this article. In particular, the classification of harmonic morphisms with a given domain Riemannian manifold $(M, g)$ can be reduced to the classification of conformal foliations on domains in $M$ that either have codimension 2 and minimal leaves or else have codimension $n \neq 2$ and satisfy (5). 


\section{Harmonic morphisms of corank 1: local theory.}

The first result of this section will be Theorem 1, which says, roughly, that the local harmonic morphisms with a specified Riemannian $n$-manifold $(N, h)$ as range depend on one arbitrary function of $n+1$ variables. The second result will be Theorem 2 , which says, roughly, that, for a specified Riemannian $(n+1)$-manifold $(M, g)$ with $n \geq 3$, the harmonic morphisms with domain $M$ form a finite dimensional space of dimension at most $\left(\begin{array}{c}n+3 \\ 2\end{array}\right)-1$. In $\S 3$, this space will be computed explicitly when $g$ is a metric of constant sectional curvature on $M$.

Since the assumption $p=1$ will hold for nearly all of the remainder of this article, it seems advisable to introduce a notational simplification: Instead of letting the index range run from 1 to $n+1$, let it run from 0 to $n$, i.e., make ' 0 ' an indicial synonym for ' $n+1$ '.

\subsection{A local normal form.}

All harmonic morphisms of corank one can be put into a simple local normal form.

Theorem 1. Let $\pi: P^{n+1} \rightarrow N^{n}$ be a principal $G$-bundle with $G=\mathbb{R}$ or $S^{1}$, let $\psi$ be a connection form on $P$, let $h$ be a Riemannian metric on $N$, and let $r$ be a positive function defined on an open set $M \subset P$. Then, when $M$ is endowed with the metric $g=r^{-2} \pi^{*}(h)+r^{2 n-4} \psi^{2}$, the projection $\pi: M \rightarrow N$ is a harmonic morphism. Moreover, every submersive harmonic morphism of corank 1 is locally of this form.

Proof. That $\pi: M \rightarrow N$ as described will be a harmonic morphism for any choice of the data $(h, \psi, r)$ is verified by a local calculation using the formulae of $\S 1.1$. I leave this to the reader, though the reason that the formula works will become clearer after the converse part of the Proposition is argued.

It remains to prove that the given local normal form holds for all submersive harmonic morphisms of corank 1 . Thus, let $\phi:\left(M^{n+1}, g\right) \rightarrow\left(N^{n}, h\right)$ be a submersion of Riemannian manifolds which is also a harmonic morphism. Choose a point $x \in M$ and let $V \subset N$ be a contractible open neighborhood of $\phi(x)=y$. Let $U \subset \phi^{-1}(V) \subset M$ be an open neighborhood of $x$ on which the line bundle $\operatorname{ker} \phi^{\prime}$ is trivial and for which the fibers of $\phi: U \rightarrow V$ are connected and simply connected. Define $r>0$ by the equation $n r^{2}=\left\|\phi^{\prime}\right\|^{2}$. 
Let $\eta_{1}, \ldots, \eta_{n}$ be an $h$-orthonormal coframing on $V$ and let $\omega_{0}$ on $U$ be chosen so that

$$
\omega_{0}, \omega_{1}=r^{-1} \phi^{*}\left(\eta_{1}\right), \ldots, \omega_{n}=r^{-1} \phi^{*}\left(\eta_{n}\right)
$$

is a $g$-orthonormal coframing of $U$, as was discussed in $\S 1.1$.

Keep the notation of $\S 1.1 .3$. Since $\phi$ is a harmonic morphism, by (13) of that section,

$$
H_{i \alpha \alpha}=H_{i 00}=-(n-2) r_{i} .
$$

Now, by equation $\left(10^{\prime}\right)$ in $\S 1.1$, there exist functions $a_{i j}=-a_{j i}$ (which would have been written as $a_{i j 0}$ in $\left.\S 1.1\right)$ and $r_{0}, r_{1}, \ldots, r_{n}$ so that

$$
\begin{aligned}
d r & =r\left(r_{0} \omega_{0}+r_{1} \omega_{1}+\cdots+r_{n} \omega_{n}\right), \\
\omega_{i 0} & =-r_{0} \omega_{i}+a_{i j} \omega_{j}-(n-2) r_{i} \omega_{0} .
\end{aligned}
$$

The structure equations then yield

$$
\begin{aligned}
d\left(r^{2-n} \omega_{0}\right) & =(2-n) r^{-1} d r \wedge r^{2-n} \omega_{0}+r^{2-n} d \omega_{0}, \\
& =r^{2-n}\left((2-n)\left(r_{a} \omega_{a}\right) \wedge \omega_{0}-\omega_{0 i} \wedge \omega_{i}\right), \\
& =r^{2-n}\left((2-n)\left(r_{i} \omega_{i}\right) \wedge \omega_{0}+\left(-r_{0} \omega_{i}+a_{i j} \omega_{j}-(n-2) r_{i} \omega_{0}\right) \wedge \omega_{i}\right), \\
& =-r^{2-n} a_{i j} \omega_{i} \wedge \omega_{j} .
\end{aligned}
$$

Set $\bar{\Omega}=-r^{2-n} a_{i j} \omega_{i} \wedge \omega_{j}$ and note that this is a closed 2-form which is $\phi$ semibasic. Since the fibers of $\phi$ in $U$ are connected, there exists a (closed) 2-form $\Omega$ on $V$ so that $\bar{\Omega}=\phi^{*} \Omega$. Since $V$ is contractible, there exists a 1 -form $\psi_{0}$ on $V$ so that $d \psi_{0}=\Omega$. On $P=V \times \mathbb{R}$ (with coordinate $t$ on the $\mathbb{R}$-factor), the 1 -form $\psi=d t+\psi_{0}$ represents a connection for $P$ regarded as a principal $\mathbb{R}$-bundle over $V$, with projection onto the first factor $\pi: P \rightarrow V$.

Locally, $\left(U, r^{2-n} \omega_{0}\right)$ and $(P, \psi)$ are principal $\mathbb{R}$-bundles over $V$ with connection and they have the same curvature, so they are locally gauge equivalent. Since $P$ is globally an $\mathbb{R}$-bundle over $V$ and the fibers of $\phi: U \rightarrow$ $V$ are contractible, there exists a unique diffeomorphic embedding $\tau: U \rightarrow P$ satisfying $\phi=\pi \circ \tau$ and $\tau^{*}(\psi)=r^{2-n} \omega_{0}$ as well as the ('gauge fixing') initial condition $\tau(x)=(\phi(x), 0) \in V \times \mathbb{R}=P$. Define $s>0$ on $\tau(U) \subset P$ so that $\tau^{*} s=r$. Then

$$
g_{\mid U}=\tau^{*}\left(s^{-2} \pi^{*}(h)+s^{2 n-4} \psi^{2}\right) .
$$

Thus, the theorem is proved. 
Example 1. Isometric Quotients. An important special case of this construction is the case where $r$ is constant on the fibers of $P$. In this case, let $\mathbf{X}$ be the $\pi$-vertical vector field that satisfies $\psi(\mathbf{X})=1$, i.e., $\mathbf{X}$ is the infinitesimal generator of the $G$-action. Then, since $r$ is constant on the flow lines of $\mathbf{X}$, it follows that $\mathbf{X}$ is a Killing vector field for $g$.

Conversely, suppose that $\mathbf{X}$ is a Killing vector field on $\left(M^{n+1}, g\right)$, i.e., that $\mathcal{L}_{\mathbf{X}} g=0$. Let $U \subset M$ be any open set on which $\mathbf{X}$ is non-vanishing and on which the foliation $\mathcal{F}_{\mathbf{X}}$ by integral curves of $\mathbf{X}$ is amenable, with submersive leaf projection $\pi: U \rightarrow N^{n}$.

Let $\psi$ be the 1 -form that is $g$-dual to $\mathbf{X}$ and let $r>0$ be defined so that $\omega_{0}=r^{n-2} \psi$ is a unit 1-form. Because $\mathbf{X}$ is a symmetry vector field for $g$, it follows that $\mathcal{L}_{\mathbf{X}} \psi=\mathcal{L}_{\mathbf{X}} r=0$, so in particular, $\mathcal{L}_{\mathbf{X}} \omega_{0}=0$. Moreover, since $\psi(\mathbf{X})=1$, it follows that $\psi$ is a connection form for $U$ regarded as a local principal $\mathbb{R}$-bundle over $N$.

Set $g^{\prime}=g-\omega_{0}{ }^{2}$, as consistent with previous usage. Since $\mathcal{L}_{\mathbf{X}} g=0$, it follows that $\mathcal{L}_{\mathbf{X}} g^{\prime}=0$. In particular, there exists a metric $h$ on $N^{n}$ so that $\pi^{*}(h)=r^{2} g^{\prime}$. (Note the use of $\mathcal{L}_{\mathbf{X}} r=0$.) Since, by construction,

$$
g=r^{-2} \pi^{*}(h)+r^{2 n-4} \psi^{2},
$$

it follows from Theorem 1 that the projection $\pi:(U, g) \rightarrow(N, h)$ is a harmonic morphism.

In this way, every non-zero Killing vector field defines a local harmonic morphism of corank 1 . Note, however, that not every harmonic morphism of corank 1 arises in this way.

Remark: Local generality. With Theorem 1 in hand, it can now be explained what is mean by the statement, "Up to local diffeomorphism, the harmonic morphisms of corank 1 depend on one arbitrary function of $(n+1)$ variables."

Consider the data $(h, \psi, r)$ described in Theorem 1 . The first two components are locally defined on an $n$-manifold and thus, up to diffeomorphism, are specified by a certain number of functions of $n$ variables. However, $r>0$ is essentially arbitrary on an open set of dimension $(n+1)$. This arbitrary function cannot be 'gauged away' by reparametrizing $M$ since $\phi$ induces on $M$ the local structure of a principal $\mathbb{R}$-bundle over an $n$-dimensional base, and the diffeomorphisms that preserve this local bundle structure depend only on functions of $n$ variables.

This sort of 'dependency' discussion can be made more precise in several ways. One way is through the language of exterior differential systems [BCG], but it can also be interpreted in terms of a formula for the 
dimension of the space of $k$-jets of metrics on a neighborhood of $\mathbf{0} \in \mathbb{R}^{n+1}$ that admit a submersive harmonic morphism to some $n$-manifold, as in the theory of Spencer, Goldschmidt, and Malgrange. However, no essential use of this notion of dependency or generality will be made in the rest of this article, so making it precise does not appear to be important. Rather, this notion of local generality will be used in a heuristic way for motivational purposes.

Remark: Morphisms with a given range metric. By Theorem 1, any Riemannian $n$-manifold $N$ can be the range of a submersive harmonic morphism of corank 1 in infinitely many distinct ways. In fact, by judicious choice of $r$, one can even arrange that the domain $\left(M^{n+1}, g\right)$ be a complete Riemannian manifold, regardless of whether $(N, h)$ is complete or not.

Remark: Higher corank. There is a local structure theorem similar to Theorem 1 for submersive harmonic morphisms of higher corank as well, but it is not quite as satisfactory.

Using the 'volume preserving' characterization mentioned at the end of $\S 1.1$, one can show that if $\phi:\left(M^{n+p}, g\right) \rightarrow\left(N^{n}, h\right)$ is a harmonic morphism and $m \in M$ is a point where $\phi^{\prime}(m) \neq 0$, then there exists an $m$ neighborhood $U \subset M$ together with coordinates $x_{1}, \ldots, x_{n+p}$ on $U$ so that the following properties hold:

(1) The fibers of $\phi$ in $U$ are defined by the equations $d x_{i}=0$.

(2) There exist functions $h_{i j}=h_{j i}$ of the variables $x_{i}$ so that

$$
\phi^{*}(h)=h_{i j} d x_{i} \circ d x_{j} .
$$

(3) There exist functions $R>0, P_{\alpha i}$, and $g_{\alpha \beta}=g_{\beta \alpha}$ of all the variables $x_{a}$ satisfying

$$
\sum_{\alpha} \frac{\partial P_{\alpha i}}{\partial x_{\alpha}}=0 \text { for all } i, \text { and } \operatorname{det}\left(g_{\alpha \beta}\right)=1
$$

so that

$$
g=R^{-p}\left(h_{i j} d x_{i} \circ d x_{j}\right)+R^{n-2} g_{\alpha \beta}\left(d x_{\alpha}+P_{\alpha i} d x_{i}\right) \circ\left(d x_{\beta}+P_{\beta j} d x_{j}\right) .
$$

The functions $x_{i}$ can be regarded as coordinates on a neighborhood of $\phi(m) \in N$. In these coordinates, $\phi$ is just the natural submersion $\mathbb{R}^{n+p} \rightarrow$ $\mathbb{R}^{n}$ given by projection on the first $n$ coordinates. 
Conversely, if $h_{i j}, R>0, P_{\alpha i}$, and $g_{\alpha \beta}$ are functions on some open set $U \subset \mathbb{R}^{n+p}$ that satisfy the conditions in (2) and (3) and, moreover, the condition that the symmetric matrices $\left(h_{i j}\right)$ and $\left(g_{\alpha \beta}\right)$ are positive definite at every point of $U$, then the metrics $g$ on $U$ and $h$ on $V=\phi(U) \subset \mathbb{R}^{n}$ defined by the above formulae have the property that $\phi:(U, g) \rightarrow(V, h)$ is a harmonic morphism.

Note that the functions $R$ and $g_{\alpha \beta}=g_{\beta \alpha}$ are 'arbitrary', being only subject to algebraic inequalities and the single algebraic equation $\operatorname{det}\left(g_{\alpha \beta}\right)=$ 1 , while the $n p$ functions $P_{\alpha i}$ are subject to $n$ linear, constant coefficient, first order PDE, but are otherwise arbitrary.

Thus, this normal form gives a local recipe for the general harmonic morphism $\phi:\left(M^{n+p}, g\right) \rightarrow\left(N^{n}, h\right)$ that depends on the choice of

$$
1+\left(\left(\begin{array}{c}
p+1 \\
2
\end{array}\right)-1\right)+n p-n=\left(\begin{array}{c}
p+1 \\
2
\end{array}\right)+n(p-1)
$$

arbitrary functions of $n+p$ variables. However, it can be shown that the ambiguity in choosing the 'normal coordinates' $x_{a}$ depends on $p-1$ arbitrary functions of $n+p$ variables. Thus, this naïve counting suggests that, up to local diffeomorphism, the general harmonic morphism $\phi:\left(M^{n+p}, g\right) \rightarrow$ $\left(N^{n}, h\right)$ depends on

$$
\left(\begin{array}{c}
p+1 \\
2
\end{array}\right)+(n-1)(p-1)
$$

arbitrary functions of $n+p$ variables. This count can be made rigorous, but it will not be necessary to do so in this article. The main point of these observations is that they indicate that, since, up to diffeomorphism, the general metric in dimension $n+p$ depends on $\left(\begin{array}{c}n+p \\ 2\end{array}\right)$ functions of $n+p$ variables rather than $\left(\begin{array}{c}p+1 \\ 2\end{array}\right)$ functions of $n+p$ variables, the general metric in this dimension will not admit, even locally, a harmonic morphism of corank $p$ except when $n=1$.

This normal form also indicates that, while any Riemannian manifold $(N, h)$ can be the range of a harmonic morphism of arbitrary corank (in fact, in infinitely many ways), the problem of finding the harmonic morphisms with a given Riemannian domain metric will involve the study of overdetermined systems and their integrability conditions.

\subsection{Given domain metric.}

While Theorem 1 does give a local normal form for harmonic morphisms of corank 1 , it does not give much effective help for solving the problem 
of determining, for a specific Riemannian manifold $\left(M^{n+1}, g\right)$, what are the possible harmonic morphisms of the form $\phi: M \rightarrow N$, where the $n$ manifold $N$ and its metric $h$ may or may not be specified in advance. This is essentially a question of when a given metric $g$ can be written locally in the normal form given by Theorem 1 and, if so, in how many ways.

2.2.1. The low dimensional cases. The case $n=1$ is basically trivial, and will not be discussed any further.

The case $n=2$ is more interesting, but has a very different character from the cases where $n \geq 3$. This is because, using the conformal flatness of metrics in dimension 2 , the local normal form can be simplified when $n=2$ to the coordinate form

$$
g=R\left(x_{1}, x_{2}, x_{3}\right)\left(d x_{1}^{2}+d x_{2}^{2}\right)+\left(d x_{3}+a\left(x_{1}, x_{2}\right) d x_{1}+b\left(x_{1}, x_{2}\right) d x_{2}\right)^{2} .
$$

where $R>0$ is an arbitrary function of three variables while $a$ and $b$ are arbitrary functions of two variables and the fibers of the harmonic morphism $\phi$ are described by the equations $d x_{1}=d x_{2}=0$.

Computation using this form shows that, at any point in $M$, the sectional curvatures of the 2-planes passing through that point and containing the tangent to the $\phi$-fiber must all be equal.

However, elementary algebra shows that, if the sectional curvature is not constant on the 2-planes through a point $x \in M$, then there are at most two tangent directions $v \in T_{x} M$ so that the sectional curvature is constant on all the 2-planes containing $v$. Thus, for a metric $g$ on $M^{3}$ of non-constant sectional curvature, there are at most two distinct foliations by curves which could be the fibers of a harmonic morphism of corank 1 .

It then suffices to apply the criteria developed in $\S 1.2$ to these two foliations (or one foliation when there are only two distinct eigenvalues of the sectional curvature at every point) to determine whether or not the given metric $g$ admits a harmonic morphism of corank 1. This has been carried out by Baird and Wood [BW2] in order to explicitly determine the metrics for which both of these foliations are the fibers of (possibly local) harmonic morphisms.

Finally, when $g$ has constant sectional curvature, there are many harmonic morphisms $\phi: U \rightarrow N^{2}$ of corank 1 with domain an open subset $U \subset M$. The local description is very simple: The fibers of such a map $\phi$ must be geodesics (since they are minimal) and so $N^{2}$ can be identified with a surface $\Sigma_{\phi}$ in the space $\Lambda(M)$ of geodesics in $M$. If $M$ is complete and simply connected, this latter space has dimension 4 and carries a canonical 
complex structure which makes it into a complex surface. The surface $\Sigma_{\phi}$ is then seen to be a complex curve in this complex surface. Conversely, if $C \subset \Lambda(M)$ is any complex curve (aside from a few special cases), then the 2parameter family of geodesics that it represents will cover an open set in $M$. By suitably restricting the curve $C$, one can arrive at an open set $U \subset M$ that is foliated by these geodesics and this foliation will be amenable and satisfy the conditions of $\S 1.2$, so that it gives rise to a harmonic morphism with domain $U$ and of corank 1 . For more details and a study of the global and singularity issues, see [BW1].

2.2.2. The high dimensional cases. The rest of this article concerns only the case $n \geq 3$. The main result to conclude this section will be a general 'finiteness' theorem, showing that the set of harmonic morphisms of corank 1 with a given Riemannian domain $\left(M^{n+1}, g\right)$ is a finite dimensional space.

Let $(M, g)$ be a Riemannian $(n+1)$-manifold. By Proposition 2, for any connected open set $U \subset M$ on which there exists a submersive harmonic morphism $\phi:(U, g) \rightarrow\left(N^{n}, h\right)$, the $n$-manifold $N$ and, up to a constant scale factor, the metric $h$ are determined by the foliation $\mathcal{F}_{\phi}$ defined by the fibers of $\phi$. For simplicity, I am going to assume that it is possible smoothly to orient the fibers of $\phi$, as can always be arranged by passing to a double cover of $U$ if necessary. Then there will exist a unique unit vector field $\mathbf{u}_{\phi}$ on $U$ tangent to the fibers of $\phi$ and inducing the given orientation on each fiber.

Conversely, given a unit vector field $\mathbf{u}$ on an open set $U \subset M$, this process can be reversed. Provided that $\mathbf{u}$ satisfies certain equations (to be spelled out below) and provided that the foliation $\mathcal{F}_{\mathbf{u}}$ of $U$ induced by the integral curves of $\mathbf{u}$ is amenable, then, by Proposition 2, there will be a metric $h$, unique up to a constant multiple, on $U / \mathcal{F}_{\mathbf{u}}$ so that the leaf projection $\phi: U \rightarrow U / \mathcal{F}_{\mathbf{u}}$ is a harmonic morphism.

The conditions on $\mathbf{u}$ needed to construct this harmonic morphism are as follows: Let $\omega_{0}$ be the 1 -form on $U$ that is $g$-dual to $\mathbf{u}$. Set $g^{\prime}=g-\omega_{0}^{2}$. Then the first condition on $\mathbf{u}$ is that the foliation $\mathcal{F}_{\mathbf{u}}$ should be conformal, i.e., that there should exist a function $r_{0}$ on $U$ so that

$$
\mathcal{L}_{\mathbf{u}}\left(g^{\prime}\right)=-2 r_{0} g^{\prime}
$$

Note that (4) is an overdetermined set of first order PDE for $\mathbf{u}$. The 1form $\rho^{\prime \prime}=r_{0} \omega_{0}$ is the same as the one constructed in $\S 1.2 .1$. Next, define a 
1-form $\rho^{\prime}$ on $U$ by the equation

$$
\mathcal{L}_{\mathbf{u}} \omega_{0}=(n-2) \rho^{\prime} .
$$

Since $n \neq 2$, this can always be done. This $\rho^{\prime}$ is the same as the one constructed in $\S 1.2 .2$.

The final condition, corresponding to (5) of $\S 1.2 .2$, is

$$
d\left(r_{0} \omega_{0}+\rho^{\prime}\right)=0 .
$$

Note that (5) is an overdetermined set of second order PDE for $\mathbf{u}$.

Conversely, by Proposition 2, conditions (4) and (5) are sufficient to imply that, locally, the integral curves of $\mathbf{u}$ are the fibers of a harmonic morphism.

To state the next theorem precisely requires the use of the language of jets. Let $S M \rightarrow M$ denote the unit sphere bundle of $M$. This is a bundle of fiber rank $n$ over $M$.

Let $J^{1}(M, S M)$ denote the bundle of 1-jets of sections of $S M$, and let $\Sigma_{1} \subset J^{1}(M, S M)$ denote the subset consisting of those 1-jets of sections that satisfy (4). (This makes sense since (4) is a set of first order equations for a section $\mathbf{u}$ of $S M$.) Then $\Sigma_{1}$ is a smooth manifold of codimension $\left(\begin{array}{c}n+1 \\ 2\end{array}\right)-1$ in $J^{1}(M, S M)$ and the basepoint projection $\Sigma_{1} \rightarrow M$ makes $\Sigma_{1}$ into a smooth bundle over $M$ with fibers of dimension $\left(\begin{array}{c}n+2 \\ 2\end{array}\right)$.

Finally, let $J^{2}(M, S M)$ denote the bundle of 2-jets of sections of $S M$, and let $\Sigma_{2} \subset J^{2}(M, S M)$ denote the subset consisting of those 2-jets of sections that satisfy (4) and (5). As will come out in the proof of Theorem 2 below, $\Sigma_{2}$ is a smooth manifold and the natural projection $\Sigma_{2} \rightarrow \Sigma_{1}$ is a smooth submersion with fibers diffeomorphic to $\mathbb{R}^{n+1}$. Thus, $\Sigma_{2}$ is a smooth bundle over $M$ with fibers of dimension

$$
(n+1)+\left(\begin{array}{c}
n+2 \\
2
\end{array}\right)=\left(\begin{array}{c}
n+3 \\
2
\end{array}\right)-1
$$

Theorem 2. The bundle $\Sigma_{2} \rightarrow M$ has a horizontal plane field $H$ of dimension $n+1$ with the property that, for every unit vector field $\mathbf{u}$ satisfying (4) and (5), the section $j^{2} \mathbf{u}$ of $\Sigma_{2}$ is tangent to $H$.

In particular, if, on a connected open set $U \subset M$, there are two unit vector fields $\mathbf{u}_{1}$ and $\mathbf{u}_{2}$, each of which satisfies (4) and (5), that agree to second order at some point of $U$, then $\mathbf{u}_{1}=\mathbf{u}_{2}$ throughout $U$.

Remark. Because the following proof is rather long, a few remarks on its structure may be in order, especially for the benefit of those readers who are 
not familiar with the methods of the moving frame and exterior differential systems.

The idea of the proof is simply to differentiate equations (4) and (5) for the vector field $\mathbf{u}$, introducing new variables to represent the derivatives at each stage, until one reaches the point where the the differentiated equations allow one to solve for all higher derivatives of $\mathbf{u}$. One could do this in local coordinates, of course, but the resulting equations would be quite unwieldy. Instead, I have chosen to work with the $g$-orthonormal frame bundle of $M$ and to use the canonical coframing available there. This has its own apparent disadvantage. Namely, one must carry the action of $\mathrm{O}(n)$ along through all of the calculations. This 'disadvantage' is actually helpful, though, since the principle that all constructions must be $\mathrm{O}(n)$-equivariant serves as a useful guide to organizing the calculations.

In the first few paragraphs of the proof, I recall the basic notation of the $g$-orthonormal frame bundle $F$. I then use $F$ to construct a bundle $X$ with base space $J^{1}(M, S M)$ on which the differential equations (4) and (5) can be expressed as the vanishing of a certain differential ideal $\mathcal{I}$. This move is the fundamental first step in converting a differential equation into an exterior differential system. Once this step is complete, the rest of the proof is really a calculation using standard techniques from exteror differential systems. I test $\mathcal{I}$ for 'involutivity' by computing its space of integral elements. I find that it is not involutive and then go on to compute its first prolongation $\mathcal{I}^{(1)}$. (This computation is the exterior differential systems version of differentiating the equations (4) and (5) and examining the consequences.) I then compute the structure equations of $\mathcal{I}^{(1)}$ and find that it has at most one integral element at each point of the prolonged space $Y$. Once this is done, Theorem 2 follows just by interpreting the structure equations. However, the calculation of the integral elements of $\mathcal{I}^{(1)}$ yields more information than this. In fact, it shows that there are integral elements only at points where certain functions $F_{i j k l}$ and $S_{i j k}$ vanish identically. This can be interpreted as saying that only certain 2 -jets of sections of $S M$ can correspond to actual solutions of (4) and (5). These conditions are rather complicated for the general metric and, consequently, I do not bother to write them out in terms of covariant derivatives and curvature (although one could certainly do this). However, they can be calculated explicitly for metrics with constant sectional curvature. I do this in $\S 3$ so that Theorem 2 can be applied to derive the classification results to be found there and that are my ultimate goal. 
Proof. Let $F \rightarrow M$ be the $g$-orthonormal frame bundle of $M$. The elements of $F$ are of the form $(m ; u)=\left(m ; u_{0}, u_{1}, \ldots, u_{n}\right)$ where $m$ is a point of $M$ and $u=\left(u_{0}, u_{1}, \ldots, u_{n}\right)$ is a $g$-orthonormal basis of $T_{m} M$. Then $\pi: F \rightarrow$ $M$ is a principal right $\mathrm{O}(n+1)$-bundle over $M$, where $\pi$ is the basepoint projection and the right action by $A=\left(A_{a b}\right)$ in $\mathrm{O}(n+1)$ is the usual one:

$$
(m ; u) \cdot A=\left(m ; u_{b} A_{b a}\right) .
$$

There are canonical 1-forms $\omega_{a}$ on $F$ that satisfy

$$
\omega_{a}(v)=\pi^{\prime}(v) \cdot u_{a}
$$

for every vector $v$ in the tangent space to $F$ at $(m ; u)$. There are also the Levi-Civita connection forms $\omega_{a b}=-\omega_{b a}$ that satisfy the first structure equations

$$
d \omega_{a}=-\omega_{a b} \wedge \omega_{b}
$$

and the second structure equations

$$
\Omega_{a b}=d \omega_{a b}+\omega_{a c} \wedge \omega_{c b}=\frac{1}{2} R_{a b c d} \omega_{c} \wedge \omega_{d}
$$

where the functions $R_{a b c d}=-R_{b a c d}=-R_{a b d c}$ are well-defined on $F$ and represent components of the Riemann curvature tensor in the sense that

$$
\pi^{*}(\operatorname{Riem}(g))=R_{a b c d} \omega_{a} \otimes \omega_{b} \otimes \omega_{c} \otimes \omega_{d} .
$$

This use of the symbols $\omega_{a}$ and $\omega_{a b}$ should not be confused with the earlier usages. Previously, the forms $\omega_{a}$ and $\omega_{a b}$ were defined on an open set $U \subset M$ and relative to a coframing, i.e., a section of $F$ over $U$.

The map $\sigma: F \rightarrow S M$ defined by $\sigma(m ; u)=\left(m, u_{0}\right)$ is a submersion and makes $F$ into a principal right $\mathrm{O}(n)$-bundle over $S M$.

Let $\mathbf{u}$ be a unit vector field on $U \subset M$ and let $F_{\mathbf{u}}=\sigma^{-1}(\mathbf{u}(U)) \subset F$. Then $\pi: F_{\mathbf{u}} \rightarrow U$ is a principal right $\mathrm{O}(n)$-bundle. On $F_{\mathbf{u}}$, the 1-form $\omega_{0}$ equals $\pi^{*}\left(\mathbf{u}^{b}\right)$, where $\mathbf{u}^{b}$ is the 1 -form that is $g$-dual to $\mathbf{u}$. Therefore, on $F_{\mathbf{u}}$, the closed 2 -form $d \omega_{0}$ must be $\pi$-basic. Thus, since

$$
d \omega_{0}=-\omega_{0 i} \wedge \omega_{i}=\omega_{i 0} \wedge \omega_{i},
$$

there must be functions $A_{i j}$ and $r_{i}$ on $F_{\mathbf{u}}$ so that

$$
\omega_{i 0}=-(n-2) r_{i} \omega_{0}+A_{i j} \omega_{j} .
$$

(The coefficient $-(n-2)$ is introduced to align this formula with previously derived ones.) 
Now $g=\left(\mathbf{u}^{b}\right)^{2}+g^{\prime}$ and calculation as in (3) of $\S 1.2 .1$ shows that $\mathcal{L}_{\mathbf{u}}\left(g^{\prime}\right)=$ $-2 r_{0} g^{\prime}$ if and only if the functions $A_{i j}$ can be written in the form

$$
A_{i j}=-r_{0} \delta_{i j}+a_{i j}
$$

where $a_{i j}=-a_{j i}$. Thus, for any unit vector field $\mathbf{u}$, condition (4) is equivalent to the condition that equations of the form

$$
\omega_{i 0}=-(n-2) r_{i} \omega_{0}+\left(-r_{0} \delta_{i j}+a_{i j}\right) \omega_{j}
$$

hold on $F_{\mathbf{u}}$ for some functions $r_{a}$ and $a_{i j}=-a_{j i}$.

Because the formula $\pi^{*}(\nabla \mathbf{u})=\omega_{i 0} \otimes u_{i}$ holds, the functions $r_{a}$ and $a_{i j}$ represent the 'undetermined' derivatives of any solution $\mathbf{u}$ of (4). This suggests that the submanifold $\Sigma_{1} \subset J^{1}(M, S M)$ and its canonical contact ideal can be described by the following construction:

Regard $\mathbb{R}^{n}$ as the set of columns of real numbers of height $n$ and $\mathfrak{s o}(n)$ as the space of skew-symmetric matrices of size $n$-by- $n$. Let

$$
X=F \times \mathbb{R} \times \mathbb{R}^{n} \times \mathfrak{s o}(n)
$$

and let $\mathbf{r}_{0}: X \rightarrow \mathbb{R}, \mathbf{r}=\left(\mathbf{r}_{i}\right): X \rightarrow \mathbb{R}^{n}$, and $\mathbf{a}=\left(\mathbf{a}_{i j}\right): F \rightarrow \mathfrak{s o}(n)$ denote the projections onto the second third and fourth factors of this product, respectively.

Define 1 -forms $\boldsymbol{\theta}_{i}$ on $X$ by the formulae

$$
\boldsymbol{\theta}_{i}=\omega_{i 0}+(n-2) \mathbf{r}_{i} \omega_{0}+\left(\mathbf{r}_{0} \delta_{i j}-\mathbf{a}_{i j}\right) \omega_{j} .
$$

Let $\mathcal{I}_{0}$ be the differential ideal on $X$ generated by the 1 -forms $\boldsymbol{\theta}_{i}$.

For vector fields $\mathbf{u}$ that satisfy condition (4), the bundle $F_{\mathbf{u}} \subset F$ can be lifted up to $X$ by the mapping

$$
(m ; u) \mapsto\left((m ; u), r_{0},\left(r_{i}\right),\left(a_{i j}\right)\right)
$$

and, by construction, this lifting $F_{\mathbf{u}} \hookrightarrow X$ is an integral manifold of $\mathcal{I}_{0}$ on which the forms $\omega_{a}$ and $\omega_{i j}$ are linearly independent.

Conversely, any connected integral manifold in $X$ of the $\boldsymbol{\theta}_{i}$ on which the forms $\omega_{a}$ and $\omega_{i j}$ are linearly independent is the lift of an open subset of $F_{\mathbf{u}}$ for some unit vector field $\mathbf{u}$ satisfying (4) that is defined (though possibly multi-valued) on some open subset of $M$. This can be verified directly, but, in any case, is a standard argument in the theory of exterior differential systems. For further details, see [BCG]. 
Let $\mathrm{O}(n)$ act freely on the right on $X$ by the formula

$$
x \cdot A=\left((m ; u), \mathbf{r}_{0}, \mathbf{r}, \mathbf{a}\right) \cdot A=\left((m ; u) \cdot A, \mathbf{r}_{0}, A^{-1} \mathbf{r}, A^{-1} \mathbf{a} A\right) .
$$

for all $A \in \mathrm{O}(n)$. The $\mathrm{O}(n)$-orbits on $X$ are the Cauchy characteristics of the Pfaffian system $\mathcal{I}_{0}$ [BCG,Chapter1]. Writing $\boldsymbol{\theta}=\left(\boldsymbol{\theta}_{i}\right)$, the formula $R_{A}^{*} \boldsymbol{\theta}=$ $A^{-1} \boldsymbol{\theta}$ holds for $A \in \mathrm{O}(n)$ and the form $\boldsymbol{\theta}$ is semibasic for the quotient projection $X \rightarrow X / \mathrm{O}(n)$. Thus, there is a well-defined Pfaffian system $\overline{\mathcal{I}}_{0}$ on $X / \mathrm{O}(n)$ of rank $n$ that pulls back to $X$ to be $\mathcal{I}_{0}$. It is important to understand the geometric meaning of the quotient $X / \mathrm{O}(n)$.

Recall that, for any smooth bundle $B \rightarrow M$, the space $J^{1}(M, B)$ of 1-jets of sections of $B$ can be identified with the space of pairs of the form $(b, P)$ where $b$ is an element of $B$ and $P \subset T_{b} B$ is an $(n+1)$-plane transverse to the fiber of $B \rightarrow M$ containing $b$.

Accordingly, a map $X \rightarrow J^{1}(M, S M)$ can be defined: For every $x \in X$, let $\mathbf{P}_{x} \subset T_{x} X$ be the codimension $n$ subspace annihilated by the components of $\boldsymbol{\theta}$. The subspace $\mathbf{P}_{x}$ contains the tangents to the $\mathrm{O}(n)$-orbits and so pushes down to define a codimension $n$ plane field $\overline{\mathbf{P}}$ on $X / \mathrm{O}(n)$ that is the annihilator of the 1-forms in $\overline{\mathcal{I}}_{0}$.

Now, $\mathbf{P}_{x}$ contains the tangents to the fibers of the submersion $\sigma_{0}$ : $X \rightarrow S M$ defined by $\sigma_{0}\left((m ; u), r_{a}, a_{i j}\right)=\left(m, u_{0}\right)$, so, for every $x \in X$, there is a well-defined subspace $\sigma_{1}(x) \subset T_{\sigma_{0}(x)} S M$ that is of codimension $n$ and is transverse to the fibers of $S M \rightarrow M$. By the above identification, this defines a smooth map $\sigma_{1}: X \rightarrow J^{1}(M, S M)$. Tracing through the definitions, one finds that the image of $\sigma_{1}$ is $\Sigma_{1}$ while the fibers of $\sigma_{1}$ are the $\mathrm{O}(n)$-orbits in $X$. Thus,

$$
X / \mathrm{O}(n)=\Sigma_{1} \subset J^{1}(M, S M)
$$

and, under this identification, $\overline{\mathcal{I}}_{0}$ is just the pullback of the canonical contact system on $J^{1}(M, S M)$ to $\Sigma_{1}$. This identification explains the significance of the system $\mathcal{I}_{0}$.

Now, condition (5) on the vector field $\mathbf{u}$ corresponds to the condition

$$
d\left(r_{0} \omega_{0}+r_{i} \omega_{i}\right)=0
$$

on $F_{\mathbf{u}}$. This suggests defining a 2-form $\Theta_{0}$ on $X$ by the formula

$$
\boldsymbol{\Theta}_{0}=d\left(\mathbf{r}_{0} \omega_{0}+\mathbf{r}_{i} \omega_{i}\right) .
$$

Note that $\mathbf{r}_{0} \omega_{0}+\mathbf{r}_{i} \omega_{i}$ is semibasic for the projection $X \rightarrow X / \mathrm{O}(n)$ and invariant under the action of $\mathrm{O}(n)$. Thus, this 1 -form is the $\sigma_{1}$-pullback of 
a 1-form on $\Sigma_{1}=X / \mathrm{O}(n)$. In particular, its exterior derivative $\boldsymbol{\Theta}_{0}$ is the $\sigma_{1}$-pullback of a 2 -form on $\Sigma_{1}$.

Let $\mathcal{I}$ be the differential ideal on $X$ generated by the (closed) 2-form $\Theta_{0}$ and the 1 -forms $\boldsymbol{\theta}_{i}$. As an algebraic ideal, $\mathcal{I}$ is generated by the 1 -forms $\boldsymbol{\theta}_{i}$ and the 2 -forms $\boldsymbol{\Theta}_{0}$ and $d \boldsymbol{\theta}_{i}$. Just as for $\mathcal{I}_{0}$, the Cauchy characteristics of $\mathcal{I}$ are the orbits of the $\mathrm{O}(n)$ action on $X$. Thus, $\mathcal{I}$ is the $\sigma_{1}$-pullback of a differential ideal $\overline{\mathcal{I}}$ on $\Sigma_{1}$.

By construction, the integral manifolds of $\overline{\mathcal{I}}$ that satisfy the independence condition $\Omega_{1}=\omega_{0} \wedge \omega_{1} \wedge \ldots \wedge \omega_{n} \neq 0$ are locally the 1-jet graphs in $\Sigma_{1} \subset J^{1}(M, S M)$ of unit vector fields $\mathbf{u}$ on open domains in $M$ that satisfy (4) and (5). Thus, it is the exterior differential system $\left(\overline{\mathcal{I}}, \Omega_{1}\right)$ that must be studied in order to determine the space of solutions to (4) and (5).

For the rest of the proof, I will be applying the standard exterior differential systems techniques to $\left(\overline{\mathcal{I}}, \Omega_{1}\right)$. The reader who wants to know more about these techniques can consult [BCG,Chapter 4]. In particular, by these methods, the space $\Sigma_{2}$, i.e., the space of 2 -jets of solutions to the equations (4) and (5), is seen to be the space of integral elements of $\left(\overline{\mathcal{I}}, \Omega_{1}\right)$ on $\Sigma_{1}$. (Recall that, given an exterior differential system $\mathcal{J}$ on a manifold $M$ with an independence condition defined by some $p$-form $\Omega$ on $M$, the integral elements of $(\mathcal{J}, \Omega)$ are the $p$-planes $E \subset T_{m} M$ on which the elements of $\mathcal{J}$ vanish but on which $\Omega$ does not.)

It is more convenient to calculate on $X$ than on $X / \mathrm{O}(n)$. Because the Cauchy characteristics of $\mathcal{I}$ are swept out by the $\mathrm{O}(n)$-action on $X$, the integral manifolds of $\left(\overline{\mathcal{I}}, \Omega_{1}\right)$ on $\Sigma_{1}$ are in one-to-one correspondence with the integral manifolds of the system $\left(\mathcal{I}, \Omega_{2}\right)$ on $X$, where

$$
\Omega_{2}=\Omega_{1} \wedge\left(\omega_{12} \wedge \omega_{13} \wedge \ldots \wedge \omega_{(n-1) n}\right) .
$$

In particular, $\Sigma_{2}$ can be computed from the structure equations of $\mathcal{I}$.

A routine, albeit tedious, calculation yields structure equations

$$
\boldsymbol{\Theta}_{0} \equiv \rho_{0} \wedge \omega_{0}+\boldsymbol{\rho}_{i} \wedge \omega_{i} \bmod \boldsymbol{\theta}
$$

and

$$
d \boldsymbol{\theta}_{i} \equiv(n-2) \boldsymbol{\rho}_{i} \wedge \omega_{0}+\left(\delta_{i j} \boldsymbol{\rho}_{0}-\boldsymbol{\alpha}_{i j}\right) \wedge \omega_{j} \bmod \boldsymbol{\theta},
$$

where

$$
\begin{aligned}
\boldsymbol{\rho}_{0} & =d \mathbf{r}_{0} \\
\boldsymbol{\rho}_{i} & =d \mathbf{r}_{i}-\mathbf{r}_{j} \omega_{j i}-\left((n-1) \mathbf{r}_{0} \mathbf{r}_{i}+\mathbf{a}_{i j} \mathbf{r}_{j}\right) \omega_{0}+\left(\mathbf{r}_{0} \mathbf{a}_{i j}-\mathbf{r}_{i j}\right) \omega_{j} \\
\boldsymbol{\alpha}_{i j} & =d \mathbf{a}_{i j}-\mathbf{a}_{k j} \omega_{k i}-\mathbf{a}_{i k} \omega_{k j}-n \mathbf{r}_{0} \mathbf{a}_{i j} \omega_{0}-\mathbf{b}_{i j k} \omega_{k}
\end{aligned}
$$


and where the expressions $\mathbf{r}_{i j}=\mathbf{r}_{j i}$ and $\mathbf{b}_{i j k}$ are defined on $X$ by the equations

$$
\mathbf{b}_{i j k}=(n-2)\left(\mathbf{r}_{i} \mathbf{a}_{j k}+\mathbf{r}_{j} \mathbf{a}_{k i}+\mathbf{r}_{k} \mathbf{a}_{j i}\right)+\frac{1}{2}\left(R_{0 i j k}-R_{0 j i k}-R_{0 k i j}\right)
$$

and

$$
-(n-2) \mathbf{r}_{i j}=\delta_{i j} \mathbf{r}_{0}^{2}+\mathbf{a}_{i k} \mathbf{a}_{k j}+(n-2)^{2} \mathbf{r}_{i} \mathbf{r}_{j}+R_{i 0 j 0} .
$$

From (6) and (7), any integral element $E \subset T_{x} X$ of $\left(\mathcal{I}, \Omega_{2}\right)$ is defined by equations

$$
\begin{aligned}
\boldsymbol{\theta}_{i} & =0 \\
\boldsymbol{\rho}_{0} & =-(n-2) s_{0} \omega_{0}-s_{i} \omega_{i}, \\
\boldsymbol{\rho}_{i} & =-s_{i} \omega_{0}-s_{0} \omega_{i} \\
\boldsymbol{\alpha}_{i j} & =-s_{i} \omega_{j}+s_{j} \omega_{i}
\end{aligned}
$$

for some unique numbers $s_{a}$ (that depend on $E$ ). Conversely, for any choice of numbers $s_{a}$, equations (9) define an integral element of $\left(\mathcal{I}, \Omega_{2}\right)$ at every point of $X$.

One can now compute the Cartan characters of $\mathcal{I}$ from structure equations $(6,7)$ and conclude that $\mathcal{I}$ is not involutive. Consequently, it will be necessary to examine the first prolongation of $\left(\mathcal{I}, \Omega_{2}\right)$, i.e., the space $Y$ of integral elements of $\left(\mathcal{I}, \Omega_{2}\right)$ and its canonical differential ideal $\mathcal{I}^{(1)}$. Now, by equations (9), the space $Y$ is diffeomorphic to

$$
Y=X \times \mathbb{R} \times \mathbb{R}^{n} .
$$

Let $\mathbf{s}_{0}: Y \rightarrow \mathbb{R}$ and $\mathbf{s}=\left(\mathbf{s}_{i}\right): Y \rightarrow \mathbb{R}^{n}$ be the projections on the second and third factors respectively. The first prolongation ideal of $\mathcal{I}$ is then the ideal $\mathcal{I}^{(1)}$ on $Y$ generated by the $\boldsymbol{\theta}_{i}$ and the 1-forms $\boldsymbol{\eta}_{a}$ and $\boldsymbol{\eta}_{i j}=-\boldsymbol{\eta}_{j i}$ defined by

$$
\begin{aligned}
\boldsymbol{\eta}_{0} & =\boldsymbol{\rho}_{0}+(n-2) \mathbf{s}_{0} \omega_{0}+\mathbf{s}_{i} \omega_{i} \\
\boldsymbol{\eta}_{i} & =\boldsymbol{\rho}_{i}+\mathbf{s}_{i} \omega_{0}+\mathbf{s}_{0} \omega_{i} \\
\boldsymbol{\eta}_{i j} & =\boldsymbol{\alpha}_{i j}+\mathbf{s}_{i} \omega_{j}-\mathbf{s}_{j} \omega_{i} .
\end{aligned}
$$

If $N \subset X$ is any integral manifold of $\left(\mathcal{I}, \Omega_{2}\right)$, there will be unique functions $s_{a}$ on $N$ so that equations (9) hold (since the tangent spaces to $N$ must be integral elements of $\left.\left(\mathcal{I}, \Omega_{2}\right)\right)$. Thus, the lifting $N \hookrightarrow Y$ defined by $x \mapsto\left(x, s_{0}(x), s_{i}(x)\right)$ for $x \in N$ lifts $N$ to an integral manifold of $\mathcal{I}^{(1)}$. Conversely, every integral manifold of $\left(\mathcal{I}^{(1)}, \Omega_{2}\right)$ on $Y$ is the lift of a unique 
integral manifold of $\left(\mathcal{I}, \Omega_{2}\right)$ on $X$. Because of this one-to-one correspondence of integral manifolds, it suffices to determine the integral manifolds on $Y$.

Now, the $\mathrm{O}(n)$-action on $X$ lifts in the obvious way to an $\mathrm{O}(n)$-action on $Y$ whose orbits are the Cauchy characteristics of the system $\mathcal{I}^{(1)}$. The quotient space $Y / \mathrm{O}(n)$ is naturally identified with the set of integral elements of $\left(\overline{\mathcal{I}}, \Omega_{1}\right)$, i.e., with $\Sigma_{2} \subset J^{2}(M, S M)$. By its very construction, the second order contact system on $J^{2}(M, S M)$ pulls back to $\Sigma_{2}$ to become an exterior differential system $\overline{\mathcal{I}}^{(1)}$ which, in turn, then pulls back to $Y$ to become $\mathcal{I}^{(1)}$.

The next step is to determine the structure equations of $\mathcal{I}^{(1)}$. Now, equations (6) and (7) can be written as

$$
\Theta_{0} \equiv \boldsymbol{\eta}_{0} \wedge \omega_{0}+\boldsymbol{\eta}_{i} \wedge \omega_{i} \quad \bmod \boldsymbol{\theta}
$$

and

$$
d \boldsymbol{\theta}_{i} \equiv(n-2) \boldsymbol{\eta}_{i} \wedge \omega_{0}+\left(\delta_{i j} \boldsymbol{\eta}_{0}-\boldsymbol{\eta}_{i j}\right) \wedge \omega_{j} \bmod \boldsymbol{\theta} .
$$

Thus, $\boldsymbol{\Theta}_{0} \equiv d \boldsymbol{\theta}_{i} \equiv 0 \bmod \boldsymbol{\theta}, \boldsymbol{\eta}$. To complete the structure equations of $\mathcal{I}^{(1)}$, formulae for $d \boldsymbol{\eta}_{a}$ and $d \boldsymbol{\eta}_{i j}$ must now be computed.

Since $d \boldsymbol{\Theta}_{0}=0$, differentiating (11) and reducing modulo $\boldsymbol{\theta}, \boldsymbol{\eta}$ yields

$$
d \boldsymbol{\eta}_{0} \wedge \omega_{0}+d \boldsymbol{\eta}_{i} \wedge \omega_{i} \equiv 0 \bmod \boldsymbol{\theta}, \boldsymbol{\eta} .
$$

It follows that there exist 1 -forms $\sigma_{a}$ so that

$$
d \boldsymbol{\eta}_{0} \equiv(n-2) \boldsymbol{\sigma}_{0} \wedge \omega_{0}+\boldsymbol{\sigma}_{i} \wedge \omega_{i} \bmod \boldsymbol{\theta}, \boldsymbol{\eta},
$$

and examination of the definition of $\boldsymbol{\eta}_{0}$ reveals that

$$
\boldsymbol{\sigma}_{a} \equiv d \mathbf{s}_{a} \quad \bmod \omega_{b}, \omega_{i j}, \boldsymbol{\theta}, \boldsymbol{\eta}
$$

(A more explicit formula for $\sigma_{a}$ will not be needed in the proof.) Substituting (14) into (13) and collecting terms yields

$$
\left(d \boldsymbol{\eta}_{i}-\sigma_{i} \wedge \omega_{0}\right) \wedge \omega_{i} \equiv 0 \bmod \boldsymbol{\theta}, \boldsymbol{\eta},
$$

which implies that there exist 1-forms $\tau_{i j}=\tau_{j i}$ so that

$$
d \boldsymbol{\eta}_{i} \equiv \boldsymbol{\sigma}_{i} \wedge \omega_{0}+\tau_{i j} \wedge \omega_{j} \quad \bmod \boldsymbol{\theta}, \boldsymbol{\eta} .
$$

Now, differentiating (12) and reducing modulo $\boldsymbol{\theta}$ and $\boldsymbol{\eta}$ yields

$$
0 \equiv(n-2) d \boldsymbol{\eta}_{i} \wedge \omega_{0}+\left(\delta_{i j} d \boldsymbol{\eta}_{0}-d \boldsymbol{\eta}_{i j}\right) \wedge \omega_{j} \bmod \boldsymbol{\theta}, \boldsymbol{\eta} .
$$


Substituting (14) and (16) into (17) and rearranging then yields

$$
\begin{aligned}
0 \equiv(n-2)\left(\tau_{i j}-\delta_{i j} \sigma_{0}\right) & \wedge \omega_{j} \wedge \omega_{0} \\
& -\left(d \boldsymbol{\eta}_{i j}-\sigma_{i} \wedge \omega_{j}+\sigma_{j} \wedge \omega_{i}\right) \wedge \omega_{j} \bmod \boldsymbol{\theta}, \boldsymbol{\eta}
\end{aligned}
$$

Reducing (18) modulo $\omega_{0}$ gives

$$
0 \equiv\left(d \boldsymbol{\eta}_{i j}-\boldsymbol{\sigma}_{i} \wedge \omega_{j}+\boldsymbol{\sigma}_{j} \wedge \omega_{i}\right) \wedge \omega_{j} \bmod \boldsymbol{\theta}, \boldsymbol{\eta}, \omega_{0} .
$$

Due to the skewsymmetry in $i$ and $j$ of the expression $d \boldsymbol{\eta}_{i j}-\boldsymbol{\sigma}_{i} \wedge \omega_{j}+\boldsymbol{\sigma}_{j} \wedge \omega_{i}$, it follows that there are functions $F_{i j k l}$ with the symmetries of a Riemann curvature tensor so that

$$
d \boldsymbol{\eta}_{i j} \equiv \boldsymbol{\sigma}_{i} \wedge \omega_{j}-\sigma_{j} \wedge \omega_{i}+\frac{1}{2} F_{i j k l} \omega_{k} \wedge \omega_{l} \bmod \boldsymbol{\theta}, \boldsymbol{\eta}, \omega_{0} .
$$

Thus, there exist 1-forms $\psi_{i j}=-\psi_{j i}$ so that

(19) $d \boldsymbol{\eta}_{i j} \equiv \boldsymbol{\sigma}_{i} \wedge \omega_{j}-\boldsymbol{\sigma}_{j} \wedge \omega_{i}+(n-2) \psi_{i j} \wedge \omega_{0}+\frac{1}{2} F_{i j k l} \omega_{k} \wedge \omega_{l} \bmod \boldsymbol{\theta}, \boldsymbol{\eta}$.

Substituting (19) into (18) yields

$$
0 \equiv(n-2)\left(\tau_{i j}-\delta_{i j} \sigma_{0}+\psi_{i j}\right) \wedge \omega_{j} \wedge \omega_{0} \bmod \boldsymbol{\theta}, \boldsymbol{\eta} .
$$

By Cartan's Lemma, there must exist functions $T_{i j k}=T_{i k j}$ so that

$$
\tau_{i j}-\delta_{i j} \sigma_{0}+\psi_{i j} \equiv 2 T_{i j k} \omega_{k} \bmod \boldsymbol{\theta}, \boldsymbol{\eta}, \omega_{0} .
$$

Symmetrizing (20) in $i$ and $j$ yields

$$
\tau_{i j}-\delta_{i j} \sigma_{0} \equiv\left(T_{i j k}+T_{j i k}\right) \omega_{k} \bmod \boldsymbol{\theta}, \boldsymbol{\eta}, \omega_{0},
$$

so that there exist functions $S_{i j}=S_{j i}$ so that

$$
\tau_{i j}-\delta_{i j} \sigma_{0} \equiv S_{i j} \omega_{0}+\left(T_{i j k}+T_{j i k}\right) \omega_{k} \bmod \boldsymbol{\theta}, \boldsymbol{\eta}
$$

Thus, (16) becomes

$$
d \boldsymbol{\eta}_{i} \equiv\left(\boldsymbol{\sigma}_{i}-S_{i j} \omega_{j}\right) \wedge \omega_{0}+\sigma_{0} \wedge \omega_{i}+T_{j i k} \omega_{k} \wedge \omega_{j} \bmod \boldsymbol{\theta}, \boldsymbol{\eta} .
$$

Replacing $\sigma_{i}$ by $\sigma_{i}-S_{i j} \omega_{j}$ will not affect (14) (because of the symmetry $S_{i j}=$ $\left.S_{j i}\right)$ and will, after a modification of the functions $F_{i j k l}$ that preserves their 
symmetries, also preserve (19). However, this substitution will simplify the above equation to

$$
d \boldsymbol{\eta}_{i} \equiv \sigma_{i} \wedge \omega_{0}+\sigma_{0} \wedge \omega_{i}+T_{j i k} \omega_{k} \wedge \omega_{j} \bmod \boldsymbol{\theta}, \boldsymbol{\eta} .
$$

On the other hand, skewsymmetrizing (20) in $i$ and $j$ yields

$$
\psi_{i j} \equiv\left(T_{i j k}-T_{j i k}\right) \omega_{k} \bmod \boldsymbol{\theta}, \boldsymbol{\eta}, \omega_{0},
$$

so (19) becomes

$$
\begin{aligned}
d \boldsymbol{\eta}_{i j} \equiv \sigma_{i} \wedge \omega_{j}- & \sigma_{j} \wedge \omega_{i} \\
& +(n-2)\left(T_{i j k}-T_{j i k}\right) \omega_{k} \wedge \omega_{0}+\frac{1}{2} F_{i j k l} \omega_{k} \wedge \omega_{l} \bmod \boldsymbol{\theta}, \boldsymbol{\eta} .
\end{aligned}
$$

Writing $S_{i j k}=-S_{i k j}=T_{j i k}-T_{k i j}$ and using the fact that $T_{i j k}=T_{i k j}$, the structure equations for the ideal $\mathcal{I}^{(1)}$ take the form

$$
\left.\begin{array}{rl}
d \boldsymbol{\theta}_{0} \equiv & 0 \\
d \boldsymbol{\eta}_{0} \equiv & (n-2) \sigma_{0} \wedge \omega_{0}+\sigma_{i} \wedge \omega_{i} \\
d \boldsymbol{\eta}_{i} \equiv & \sigma_{i} \wedge \omega_{0}+\sigma_{0} \wedge \omega_{i}-\frac{1}{2} S_{i j k} \omega_{j} \wedge \omega_{k} \\
d \boldsymbol{\eta}_{i j} \equiv & \sigma_{i} \wedge \omega_{j}-\sigma_{j} \wedge \omega_{i} \\
& \quad+(n-2) S_{k i j} \omega_{k} \wedge \omega_{0}+\frac{1}{2} F_{i j k l} \omega_{k} \wedge \omega_{l}
\end{array}\right\} \bmod \boldsymbol{\theta}, \boldsymbol{\eta}
$$

where the 1 -forms $\sigma_{a}$ pull back to each fibers of $Y \rightarrow X$ to be the 1 -forms $d \mathbf{s}_{a}$.

Now, equations (21) do not uniquely determine the $\sigma_{a}$. For any functions $p_{a}$ on $Y$, replacing $\sigma_{0}$ by $\sigma_{0}+p_{a} \omega_{a}$ and $\sigma_{i}$ by $\sigma_{i}+(n-2) p_{i} \omega_{0}+p_{0} \omega_{i}$ will keep the form of (21) but will modify the functions $S_{i j k}$ and $F_{i j k l}$. In particular, the traced functions $S_{i i j}$ and $F_{i j i j}$ will be replaced by $S_{i i j}+(n-1) p_{j}$ and $F_{i j i j}+2 n(n-1) p_{0}$ respectively. It follows that there is a unique choice of the $\sigma_{a}$ so that the torsion functions satisfy the trace conditions

$$
S_{i i j}=0 \quad \text { and } \quad F_{i j i j}=0 .
$$

Henceforth, I assume that the $\sigma_{a}$ have been chosen so that (21) and (22) hold.

Now, I claim that there is at most one integral element of $\left(\mathcal{I}^{(1)}, \Omega_{2}\right)$ at each point of $Y$. In fact, any such integral element $E \subset T_{y} Y$ will be defined by equations of the form

$$
\theta_{i}=\eta_{a}=\eta_{i j}=\sigma_{0}-p_{a} \omega_{a}=\sigma_{i}-p_{i a} \omega_{a}=0
$$


for some unique numbers $p_{a}$, and $p_{i a}$. By (21), in order that $d \eta_{0}$ vanish on such an $E$, it is necessary and sufficient that

$$
p_{i 0}=(n-2) p_{i} \quad \text { and } \quad p_{i j}=p_{j i} .
$$

Moreover, again by (21), in order that $d \boldsymbol{\eta}_{i}$ also vanish on such an $E$, it is necessary and sufficient that

$$
p_{i j}=\delta_{i j} p_{0} \quad \text { and } \quad S_{i j k}(y)=\delta_{i j} p_{k}-\delta_{i k} p_{j} .
$$

However, the trace condition (22) implies that this last condition can only hold if

$$
p_{i}=0 \quad \text { and } \quad S_{i j k}(y)=0 .
$$

Thus, the defining relations for the integral element $E$ take the form

$$
\boldsymbol{\theta}_{i}=\boldsymbol{\eta}_{a}=\boldsymbol{\eta}_{i j}=\sigma_{0}-p_{0} \omega_{0}=\sigma_{i}-p_{0} \omega_{i}=0
$$

and these can only define an integral element at points $y \in Y$ where $S_{i j k}(y)=0$. Finally, for $d \boldsymbol{\eta}_{i j}$ to vanish on $E$, it is necessary and sufficient that $S_{i j k}(y)=0$ and

$$
F_{i j k l}(y)-p_{0}\left(\delta_{i k} \delta_{j l}-\delta_{i l} \delta_{j k}\right)=0 .
$$

Again, using the trace condition (22), this implies $p_{0}=0$ and $F_{i j k l}(y)=0$.

Thus, the subspace $\mathbf{Q}_{y} \subset T_{y} Y$ defined by the equations

$$
\theta_{i}=\eta_{a}=\eta_{i j}=\sigma_{0}=\sigma_{i}=0
$$

is the only possible integral element of $\left(\mathcal{I}^{(1)}, \Omega_{2}\right)$ at $y \in Y$ and this really is an integral element if and only if $y$ satisfies

$$
S_{i j k}(y)=F_{i j k l}(y)=0 .
$$

Since, at each $y \in Y$, the space $\mathbf{Q}_{y}$ contains the tangents to the $\mathrm{O}(n)$ orbit through $y$, there is a unique $(n+1)$-plane field $H \subset T \Sigma_{2}$ that pulls back via the projection $Y \rightarrow \Sigma_{2}$ to be $\mathbf{Q} \subset T Y$.

Finally, a unit vector field $\mathbf{u}$ on $U \subset M$ satisfies (4) and (5) if and only if the bundle $F_{\mathbf{u}}$ lifts to $Y$ to be an integral manifold of $\left(\mathcal{I}^{(1)}, \Omega_{2}\right)$, i.e., so that it is tangent to the plane field $\mathbf{Q}$. Since the image of this lifting under the projection $Y \rightarrow \Sigma_{2}$ is the image of the section $j^{2} \mathbf{u}$ of $\Sigma_{2}$, it follows that $\mathbf{u}$ satisfies (4) and (5) if and only if the image of $j^{2} \mathbf{u}$ is tangent to $H$. 
Remark: Interpretation. One way of interpreting Theorem 2 is as follows: All vector fields $\mathbf{u}$ that satisfy (4) and (5) satisfy a certain total differential equation of the form

$$
\nabla^{3} \mathbf{u}=\mathbb{R}\left(\mathbf{u}, \nabla \mathbf{u}, \nabla^{2} \mathbf{u}\right)
$$

where $\mathbf{R}$ is a certain nonlinear bundle map from $J^{2}(M, S M)$ to $T M \otimes$ $T^{*} M \otimes T^{*} M \otimes T^{*} M$. The formula for $\mathbf{R}$ in terms of the metric $g$ can be written out, but it is unenlightening and, moreover, not of much use for doing calculations, so I will not write it out here.

Remark: Integrability. When $n$ is sufficiently large, for the generic metric $g$, the equations $S_{i j k}=F_{i j k l}=0$ on $Y$ will have no solution in $Y$. In this case, there will be no harmonic morphism of corank 1 whose domain is an open subset of $M$.

Even when the locus $Z \subset Y$ defined by $S_{i j k}=F_{i j k l}=0$ is non-empty and smooth, it can well happen that there is no point $z \in Z$ so that $\mathbf{Q}_{z}$ is a subspace of $T_{z} Z$. Again, in this case, there will be no harmonic morphism of corank 1 whose domain is an open subset of $M$.

Even when there is a smooth submanifold $Z \subset Y$ that lies in the locus defined by $S_{i j k}=F_{i j k l}=0$ with the property that $\mathbf{Q}_{z}$ is a subspace of $T_{z} Z$ for all $z \in Z$, it can still happen that $\mathbf{Q}$ is not an integrable plane field on $Z$.

Finally, when there is a smooth submanifold $Z \subset Y$ that lies in the locus defined by $S_{i j k}=F_{i j k l}=0$ with the property that $\mathbf{Q}_{z}$ is a subspace of $T_{z} Z$ for all $z \in Z$ and that $\mathbf{Q}$ is an integrable plane field on $Z$, then $Z$ will be foliated by integral manifolds of $\mathcal{I}^{(1)}$. Each such integral manifold will correspond to a locally defined harmonic morphism on some domain in $M$.

Now, the functions $S_{i j k}$ and $F_{i j k l}$ on $Y$ can be expressed explicitly in terms of the functions $\mathbf{r}_{a}, \mathbf{a}_{i j}, \mathbf{s}_{a}$, and the Riemann curvature tensor components $R_{i j k l}$ and their first covariant derivatives on $F$. These formulae are very messy in general and it is difficult to tell much about them. However, in the case that $g$ has constant sectional curvature, these formulae simplify and constructive use can be made of them. In the next section, the system $\mathcal{I}^{(1)}$ will be examined for such metrics, resulting in a complete classification of the harmonic morphisms of corank one with Riemannian domain having constant sectional curvature. 


\section{Harmonic morphisms of corank 1 from space forms.}

In this section, $M$ will always denote a manifold of dimension $(n+1) \geq 4$ that is endowed with a metric $g$ with constant sectional curvature $K$. In this case, the structure equations on the orthonormal frame bundle $\pi: F \rightarrow M$ simplify to

$$
\begin{aligned}
d \omega_{a} & =-\omega_{a b} \wedge \omega_{b}, \\
d \omega_{a b} & =-\omega_{a c} \wedge \omega_{c b}+K \omega_{a} \wedge \omega_{b},
\end{aligned}
$$

i.e., $R_{a b c d}=K\left(\delta_{a c} \delta_{b d}-\delta_{a d} \delta_{b c}\right)$.

\subsection{Analysis of the exterior differential system.}

As in the proof of Theorem 2, define the manifold

$$
X=F \times \mathbb{R} \times \mathbb{R}^{n} \times \mathfrak{s o}(n)
$$

and let $\mathbf{r}_{0}: X \rightarrow \mathbb{R}, \mathbf{r}=\left(\mathbf{r}_{i}\right): X \rightarrow \mathbb{R}^{n}$, and $\mathbf{a}=\left(\mathbf{a}_{i j}\right): F \rightarrow \mathfrak{s o}(n)$ denote the projections onto the second third and fourth factors of this product, respectively. As before define 1-forms $\boldsymbol{\theta}_{i}$ on $X$ by the formulae

$$
\boldsymbol{\theta}_{i}=\omega_{i 0}+(n-2) \mathbf{r}_{i} \omega_{0}+\left(\mathbf{r}_{0} \delta_{i j}-\mathbf{a}_{i j}\right) \omega_{j} .
$$

Next, define

$$
Y=X \times \mathbb{R} \times \mathbb{R}^{n}
$$

and let $\mathbf{s}_{0}: Y \rightarrow \mathbb{R}$ and $\mathbf{s}=\left(\mathbf{s}_{i}\right): Y \rightarrow \mathbb{R}^{n}$ be the projections on the second and third factors respectively.

Define 1-forms $\boldsymbol{\eta}_{a}$ and $\boldsymbol{\eta}_{i j}$ via the equations (8) and (10) of $\S 2.2$. Note, however, that the defining equations for $\mathbf{r}_{i j}$ and $\mathbf{b}_{i j k}$ simplify to

$$
\begin{aligned}
\mathbf{b}_{i j k} & =(n-2)\left(\mathbf{r}_{i} \mathbf{a}_{j k}+\mathbf{r}_{j} \mathbf{a}_{k i}+\mathbf{r}_{k} \mathbf{a}_{j i}\right) \\
-(n-2) \mathbf{r}_{i j} & =\delta_{i j}\left(\mathbf{r}_{0}{ }^{2}+K\right)+\mathbf{a}_{i k} \mathbf{a}_{k j}+(n-2)^{2} \mathbf{r}_{i} \mathbf{r}_{j} .
\end{aligned}
$$

3.1.1. First torsion equations. With great effort, 1 -forms $\sigma_{a}$ and functions $S_{i j k}$ and $F_{i j k l}$ can be explicitly computed on $Y$ so that equations (21) and (22) hold. In particular, this computation yields $\left(1_{i j k}\right)$

$$
(n-2) S_{i j k}=(n-1)\left(\mathbf{p}_{j} \mathbf{a}_{k i}-\mathbf{p}_{k} \mathbf{a}_{j i}-2 \mathbf{p}_{i} \mathbf{a}_{j k}\right)-3\left(\delta_{i j} \mathbf{p}_{l} \mathbf{a}_{k l}-\delta_{i k} \mathbf{p}_{l} \mathbf{a}_{j l}\right)
$$

where

$$
\mathbf{p}_{i}=\mathbf{s}_{i}-(n-2) \mathbf{r}_{0} \mathbf{r}_{i}
$$


The formula for $F_{i j k l}$ is much more complicated. Thankfully, it will not be needed.

Let $Z \subset Y$ be the locus defined by the equations $S_{i j k}=0$. It is clear from equation (1) that $Z$ contains the two loci $Z_{1}$ and $Z_{2}$ where

$$
\begin{aligned}
& Z_{1}=\left\{z \in Y \mid \mathbf{a}_{i j}(z)=0 \text { for all } i, j\right\}, \\
& Z_{2}=\left\{z \in Y \mid \mathbf{p}_{i}(z)=0 \text { for all } i\right\} .
\end{aligned}
$$

I claim that, in fact, $Z=Z_{1} \cup Z_{2}$. To prove this, it is enough to show, for any $p=\left(p_{i}\right) \in \mathbb{R}^{n}$ and any skew-symmetric matrix $a=\left(a_{i j}\right)$, that the equations

$$
\left(1_{i j k}^{\prime}\right) \quad 0=(n-1)\left(p_{j} a_{k i}-p_{k} a_{j i}-2 p_{i} a_{j k}\right)-3\left(\delta_{i j} p_{l} a_{k l}-\delta_{i k} p_{l} a_{j l}\right)
$$

hold for all $i, j$, and $k$ only if either $p_{i}=0$ for all $i$ or else $a_{i j}=0$ for all $i$ and $j$. To show this, set $q_{i}=a_{i j} p_{j}$. Note that, because of the skewsymmetry of $a$, it follows that $q_{i} p_{i}=a_{i j} p_{j} p_{i}=0$. Multiplying $\left(1_{i j k}^{\prime}\right)$ by $p_{k}$ and summing over $k$ yields

$$
0=(n-1)\left(-p_{j} q_{i}-\left(p_{k}\right)^{2} a_{j i}-2 p_{i} q_{j}\right)+3 q_{j} p_{i}
$$

which can be rearranged to give

$$
(n-1)\left(p_{k}\right)^{2} a_{j i}=-(n-1) p_{j} q_{i}-(2 n-5) p_{i} q_{j}
$$

Symmetrizing $\left(3_{i j}\right)$ in $i$ and $j$ yields the relation $0=p_{i} q_{j}+p_{j} q_{i}$, valid for all $i$ and $j$. Multiplying this last relation by $p_{i}$ and summing over $i$ yields $\left(p_{i}\right)^{2} q_{j}=0$. Thus, unless $p_{i}=0$ for all $i$, then $q_{j}=0$ for all $j$, and, thence, by the equations $\left(3_{i j}\right)$, it would follow that $a_{i j}=0$ for all $i$ and $j$. Thus, the claim is proved.

Now, from the proof of Theorem 2, any integral manifold of $\left(\mathcal{I}^{(1)}, \Omega_{2}\right)$ in $Y$ must lie in the locus $Z=Z_{1} \cup Z_{2}$. Since all of the connected integral manifolds of the system $\mathcal{I}^{(1)}$ are derived from solutions of the harmonic morphism equations and hence must be real analytic, it follows that any connected integral manifold of $\mathcal{I}^{(1)}$ must lie in either $Z_{1}$ or $Z_{2}$ (or possibly, both). Thus, there are two types of solutions to be studied: The first type are the integral manifolds that lie in $Z_{1}$ and the second type are the integral manifolds that lie in $Z_{2}$.

3.1.2. Integral manifolds of the first type. Since $Z_{1}$ is defined by the equations $\mathbf{a}_{i j}=0$, it follows from the definitions and the simplified formula for $\mathbf{b}_{i j k}$ that, on $Z_{1}$, the formula for $\boldsymbol{\eta}_{i j}$ simplifies to

$$
\eta_{i j}=\mathrm{s}_{i} \omega_{j}-\mathrm{s}_{j} \omega_{i}
$$


In particular, any integral manifold of $\left(\mathcal{I}^{(1)}, \Omega_{2}\right)$ in $Z_{1}$ must lie in the sublocus $Z_{11} \subset Z_{1}$ defined by the equations $\mathbf{a}_{i j}=\mathbf{s}_{j}=0$.

Now, on $Z_{11}$, the ideal $\mathcal{I}^{(1)}$ is generated by the 1 -forms

$$
\begin{aligned}
\boldsymbol{\theta}_{i}= & \omega_{i 0}+(n-2) \mathbf{r}_{i} \omega_{0}+\mathbf{r}_{0} \omega_{i} \\
\eta_{0}= & d \mathbf{r}_{0}+(n-2) \mathbf{s}_{0} \omega_{0} \\
\boldsymbol{\eta}_{i}= & d \mathbf{r}_{i}-\mathbf{r}_{j} \omega_{j i}-(n-1) \mathbf{r}_{0} \mathbf{r}_{i} \omega_{0} \\
& +(n-2) \mathbf{r}_{i} \mathbf{r}_{j} \omega_{j}+\left(\mathbf{s}_{0}+\frac{1}{n-2}\left(\mathbf{r}_{0}{ }^{2}+K\right)\right) \omega_{i}
\end{aligned}
$$

and their exterior derivatives. With a slight notation change, this becomes

$$
\begin{aligned}
\boldsymbol{\theta}_{i} & =\omega_{i 0}+(n-2) \mathbf{r}_{i} \omega_{0}+\mathbf{r}_{0} \omega_{i} \\
\boldsymbol{\eta}_{0} & =d \mathbf{r}_{0}+\left((n-2) \mathbf{s}_{0}-\mathbf{r}_{0}^{2}-K\right) \omega_{0}, \\
\boldsymbol{\eta}_{i} & =d \mathbf{r}_{i}-\mathbf{r}_{j} \omega_{j i}-(n-1) \mathbf{r}_{0} \mathbf{r}_{i} \omega_{0}+(n-2) \mathbf{r}_{i} \mathbf{r}_{j} \omega_{j}+\mathbf{s}_{0} \omega_{i} .
\end{aligned}
$$

A short computation yields

$$
\left.\begin{array}{rl}
d \boldsymbol{\theta}_{i} & \equiv 0 \\
d \boldsymbol{\eta}_{0} & \equiv \boldsymbol{\sigma}_{0} \wedge \omega_{0} \\
d \boldsymbol{\eta}_{i} & \equiv \sigma_{0} \wedge \omega_{i}-2(n-1)(n-2) \mathbf{r}_{0} \mathbf{r}_{i} \mathbf{r}_{k} \omega_{k} \wedge \omega_{0}
\end{array}\right\} \bmod \boldsymbol{\theta}, \boldsymbol{\eta},
$$

where

$$
\sigma_{0}=d \mathbf{s}_{0}-\mathbf{r}_{0}\left(n \mathbf{s}_{0}+(n-2) \mathbf{r}_{k}^{2}\right) \omega_{0}+\left((n-2) \mathbf{s}_{0}-\mathbf{r}_{0}^{2}-K\right) \mathbf{r}_{k} \omega_{k}
$$

From (5) and the real analyticity mentioned before, it follows that any connected integral manifold of $\left(\mathcal{I}^{(1)}, \Omega_{2}\right)$ that lies in $Z_{11}$ must lie in either the sublocus $Z_{111} \subset Z_{11}$ defined by the equation $\mathbf{r}_{0}=0$ or the sublocus $Z_{112} \subset Z_{11}$ defined by the equations $\mathbf{r}_{i}=0$ for all $i=1, \ldots n$.

Now, pulled back to $Z_{111}$, the 1 -form $\eta_{0}$ simplifies to

$$
\eta_{0}=\left((n-2) \mathbf{s}_{0}-K\right) \omega_{0},
$$

so any integral manifold of $\left(\mathcal{I}^{(1)}, \Omega_{2}\right)$ that lies in $Z_{111}$ must lie in the sublocus $Z_{1111} \subset Z_{111}$ defined by the equation $\mathbf{s}_{0}=K /(n-2)$. Now, on $Z_{1111}$, the generators of $\mathcal{I}^{(1)}$ simplify to

$$
\begin{aligned}
\boldsymbol{\theta}_{i} & =\omega_{i 0}+(n-2) \mathbf{r}_{i} \omega_{0} \\
\boldsymbol{\eta}_{i} & =d \mathbf{r}_{i}-\mathbf{r}_{j} \omega_{j i}+(n-2) \mathbf{r}_{i} \mathbf{r}_{j} \omega_{j}+\frac{1}{(n-2)} K \omega_{i} .
\end{aligned}
$$


and these have structure equations

$$
d \boldsymbol{\theta}_{i} \equiv d \boldsymbol{\eta}_{i} \equiv 0 \bmod \boldsymbol{\theta}, \boldsymbol{\eta} .
$$

Thus, the system $\mathcal{I}^{(1)}$ on $Z_{1111}$ is a Frobenius Pfaffian system, so that $Z_{1111}$ is foliated by integral manifolds of $\left(\mathcal{I}^{(1)}, \Omega_{2}\right)$. I will call the foliations of $M$ corresponding to these integral manifolds, foliations of Type 0 . They will be analyzed below.

Now examine the system $\left(\mathcal{I}^{(1)}, \Omega_{2}\right)$ on the sublocus $Z_{112} \subset Z_{11}$. On this sublocus, the 1 -forms $\eta_{i}$ simplify to $\eta_{i}=\mathrm{s}_{0} \omega_{i}$, so all of the integral manifolds of $\left(\mathcal{I}^{(1)}, \Omega_{2}\right)$ in $Z_{112}$ must, in fact, lie in the sublocus $Z_{1121} \subset Z_{112}$ defined by the equation $\mathbf{s}_{0}=0$. On $Z_{1121}$, the system $\mathcal{I}^{(1)}$ has generators

$$
\begin{aligned}
\boldsymbol{\theta}_{i} & =\omega_{i 0}+\mathbf{r}_{0} \omega_{i}, \\
\boldsymbol{\eta}_{0} & =d \mathbf{r}_{0}-\left(\mathbf{r}_{0}^{2}+K\right) \omega_{0},
\end{aligned}
$$

and these satisfy the structure equations

$$
d \boldsymbol{\theta}_{i} \equiv d \boldsymbol{\eta}_{0} \equiv 0 \bmod \boldsymbol{\theta}, \boldsymbol{\eta}_{0} .
$$

In particular, the system $\mathcal{I}^{(1)}$ is a Frobenius Pfaffian system on $Z_{1121}$ and so it is foliated by integral manifolds of $\left(\mathcal{I}^{(1)}, \Omega_{2}\right)$. I will call the foliations of $M$ corresponding to these integral manifolds, foliations of Type 1. They will be analyzed below.

3.1.3. Integral manifolds of the second type. Now consider the locus $Z_{2} \subset Z$ defined by the equations $\mathbf{s}_{i}=(n-2) \mathbf{r}_{0} \mathbf{r}_{i}$. Calculation now shows that on $Z_{2}$, the relation

$$
d \boldsymbol{\eta}_{0} \equiv(n-2) \boldsymbol{\sigma}_{0} \wedge \omega_{0}-(n-2)\left(\mathbf{s}_{0}-\mathbf{r}_{0}^{2}\right) \mathbf{a}_{i j} \omega_{i} \wedge \omega_{j} \bmod \boldsymbol{\theta}, \boldsymbol{\eta} .
$$

holds, where $\sigma_{0} \equiv d \mathbf{s}_{0} \bmod \omega$. It follows that any integral manifold of $\left(\mathcal{I}^{(1)}, \Omega_{2}\right)$ in $Z_{2}$ must lie in either the sublocus $Z_{21} \subset Z_{2}$ defined by the equation $\mathrm{s}-\mathrm{r}_{0}^{2}=0$ or else in the sublocus $Z_{1} \cap Z_{2} \subset Z_{2}$ defined by the equations $\mathbf{a}_{i j}=0$. Since all of the integral manifolds of $\left(\mathcal{I}^{(1)}, \Omega_{2}\right)$ that lie in $Z_{1}$ have already been found, this second case will be discarded.

Thus, consider the system $\left(\mathcal{I}^{(1)}, \Omega_{2}\right)$ on $Z_{21}$. The 1 -form generators of this system are

$$
\begin{aligned}
\boldsymbol{\theta}_{i}= & \omega_{i 0}+(n-2) \mathbf{r}_{i} \omega_{0}+\left(\mathbf{r}_{0} \delta_{i j}-\mathbf{a}_{i j}\right) \omega_{j} \\
\boldsymbol{\eta}_{0}= & d \mathbf{r}_{0}+(n-2) \mathbf{r}_{0}\left(\mathbf{r}_{0} \omega_{0}+\mathbf{r}_{i} \omega_{i}\right) \\
\boldsymbol{\eta}_{i}= & d \mathbf{r}_{i}-\mathbf{r}_{j} \omega_{j i}-\left(\mathbf{r}_{0} \mathbf{r}_{i}-\mathbf{a}_{i j} \mathbf{r}_{j}\right) \omega_{0}+\left(\mathbf{r}_{0} \mathbf{a}_{i j}-\mathbf{r}_{i j}+\mathbf{r}_{0}^{2} \delta_{i j}\right) \omega_{j} \\
\boldsymbol{\eta}_{i j}= & d \mathbf{a}_{i j}-\mathbf{a}_{k j} \omega_{k i}-\mathbf{a}_{i k} \omega_{k j} \\
& \quad-n \mathbf{r}_{0} \mathbf{a}_{i j} \omega_{0}-(n-2)\left(\mathbf{b}_{i j k}-\mathbf{r}_{0} \mathbf{r}_{i} \delta_{j k}+\mathbf{r}_{0} \mathbf{r}_{j} \delta_{i k}\right) \omega_{k}
\end{aligned}
$$


where, for simplicity, $\mathbf{b}_{i j k}$ has been redefined to be

$$
\mathbf{b}_{i j k}=\mathbf{r}_{i} \mathbf{a}_{j k}+\mathbf{r}_{j} \mathbf{a}_{k i}+\mathbf{r}_{k} \mathbf{a}_{j i} .
$$

On $Z_{21}$, the congruences $d \boldsymbol{\theta}_{i} \equiv d \boldsymbol{\eta}_{0} \equiv 0 \bmod \boldsymbol{\theta}, \boldsymbol{\eta}$ hold, but

$$
d \boldsymbol{\eta}_{i} \equiv 2(n-1) \mathbf{r}_{0}^{2} \omega_{i} \wedge\left(\mathbf{r}_{k} \omega_{k}\right) \quad \bmod \omega_{0}, \boldsymbol{\theta}, \boldsymbol{\eta} .
$$

It follows that any connected integral manifold of $\left(\mathcal{I}^{(1)}, \Omega_{2}\right)$ that lies in $Z_{21}$ must lie in either the sublocus $Z_{211} \subset Z_{21}$ defined by the equation $\mathbf{r}_{0}=0$ or the sublocus $Z_{212} \subset Z_{21}$ defined by the equations $\mathbf{r}_{i}=0$.

On $Z_{211}$, the generators of $\mathcal{I}^{(1)}$ simplify to

$$
\begin{aligned}
\boldsymbol{\theta}_{i} & =\omega_{i 0}+(n-2) \mathbf{r}_{i} \omega_{0}-\mathbf{a}_{i j} \omega_{j} \\
\boldsymbol{\eta}_{i} & =d \mathbf{r}_{i}-\mathbf{r}_{j} \omega_{j i}+\mathbf{a}_{i j} \mathbf{r}_{j} \omega_{0}-\mathbf{r}_{i j} \omega_{j} \\
\boldsymbol{\eta}_{i j} & =d \mathbf{a}_{i j}-\mathbf{a}_{k j} \omega_{k i}-\mathbf{a}_{i k} \omega_{k j}-(n-2)\left(\mathbf{r}_{i} \mathbf{a}_{j k}+\mathbf{r}_{j} \mathbf{a}_{k i}+\mathbf{r}_{k} \mathbf{a}_{j i}\right) \omega_{k}
\end{aligned}
$$

where

$$
-(n-2) \mathbf{r}_{i j}=K \delta_{i j}+\mathbf{a}_{i k} \mathbf{a}_{k j}+(n-2)^{2} \mathbf{r}_{i} \mathbf{r}_{j}
$$

Now computation yields

$$
d \boldsymbol{\theta}_{i} \equiv d \boldsymbol{\eta}_{i} \equiv d \boldsymbol{\eta}_{i j} \equiv 0 \bmod \boldsymbol{\theta}, \boldsymbol{\eta} .
$$

Thus, on $Z_{211}$, the system $\mathcal{I}^{(1)}$ is a Frobenius Pfaffian system. It follows that $Z_{211}$ is foliated by integral manifolds of $\left(\mathcal{I}^{(1)}, \Omega_{2}\right)$. I will call the foliations of $M$ corresponding to these integral manifolds, foliations of Type 2. Note that $Z_{1111}$ is a submanifold of $Z_{211}$ and that the integral manifolds of Type 0 are special cases of those of Type 2 . They will be analyzed below.

Finally, consider the system $\mathcal{I}^{(1)}$ on the locus $Z_{212}$. On this submanifold, the formula for $\boldsymbol{\eta}_{i}$ simplifies to

$$
\eta_{i}=\left(\mathbf{r}_{0} \mathbf{a}_{i j}-\mathbf{r}_{i j}+\mathbf{r}_{0}^{2} \delta_{i j}\right) \omega_{j}
$$

Since $\mathbf{r}_{i j}=\mathbf{r}_{j i}$, the vanishing of $\boldsymbol{\eta}_{i}$ on a connected integral manifold of $\left(\mathcal{I}^{(1)}, \Omega_{2}\right)$ that lies in $Z_{212}$ implies that the functions $\mathbf{r}_{0} \mathbf{a}_{i j}$ must also vanish on such an integral manifold. Thus, such an integral manifold must either satisfy $\mathbf{r}_{0}=0$, in which case, it lies in $Z_{211}$ and so has already been accounted for, or else satisfy $\mathbf{a}_{i j}=0$, in which case, it lies in $Z_{1}$ and so has already been accounted for. 
3.1.4. Conclusions. In summary, there are two types of integral manifolds of $\left(\mathcal{I}^{(1)}, \Omega_{2}\right)$ : When $\mathcal{I}^{(1)}$ is pulled back to either $Z_{1121}$ or $Z_{211}$, it becomes a Frobenius system whose leaves are maximal integral manifolds of $\left(\mathcal{I}^{(1)}, \Omega_{2}\right)$. Moreover every connected integral manifold of $\left(\mathcal{I}^{(1)}, \Omega_{2}\right)$ is an open subset of one of these leaves.

\subsection{The local classification.}

Finally, the main result can be stated and proved. Note that this theorem does not require any assumption of compactness or completeness. The reader is reminded that the assumption $n \geq 3$ remains in force, here and for the remainder of the article.

Theorem 3. Let $\left(M^{n+1}, g\right)$ be a 1-connected manifold of constant sectional curvature $K$ and suppose that $\phi:\left(M^{n+1}, g\right) \rightarrow\left(N^{n}, h\right)$ be a submersive harmonic morphism with connected fibers. Then either there exists a Killing field $\mathbf{X}$ tangent to the fibers of $\phi$ or else the fibers of $\phi$ are geodesics and $M$ is foliated by totally umbilic hypersurfaces that are orthogonal to the fibers of $\phi$.

Proof. Since $M$ is 1-connected and the fibers of $\phi$ are connected, it follows that the fibers are orientable, i.e., that there exists a unit vector field $\mathbf{u}$ on $M$ whose integral curves are the fibers of $\phi$. Using the notation established in. the proof of Theorem 2, let $F_{\mathbf{u}} \subset F$ be the subbundle of the $g$-orthonormal frame bundle of $M$ consisting of those frames $(m ; u)$ so that $u_{0}=\mathbf{u}(m)$.

Then there exist functions $r_{0}, r_{i}$, and $a_{i j}=-a_{j i}$ on $F_{\mathbf{u}}$ so that

$$
\omega_{i 0}=-(n-2) r_{i} \omega_{0}-\left(r_{0} \delta_{i j}-a_{i j}\right) \omega_{j} .
$$

Moreover, these functions satisfy

$$
d\left(r_{0} \omega_{0}+r_{i} \omega_{i}\right)=0 .
$$

By the analysis in $\S 3.1$, there are two possibilities. Either $r_{i}=a_{i j}=0$ and $d r_{0}=\left(r_{0}^{2}+K\right) \omega_{0}$ (if the corresponding integral manifold is of Type 1) or else $r_{0}=0$ and the functions $r_{i}$ and $a_{i j}$ satisfy the equations

$$
\begin{aligned}
d r_{i} & =r_{j} \omega_{j i}-a_{i j} r_{j} \omega_{0}+r_{i j} \omega_{j}, \\
d a_{i j} & =a_{k j} \omega_{k i}+a_{i k} \omega_{k j}+(n-2)\left(r_{i} a_{j k}+r_{j} a_{k i}+r_{k} a_{j i}\right) \omega_{k},
\end{aligned}
$$

where

$$
-(n-2) r_{i j}=K \delta_{i j}+a_{i k} a_{k j}+(n-2)^{2} r_{i} r_{j}
$$


(if the corresponding integral manifold is of Type 2).

Suppose that the first possibility holds. Then, since $r_{i}=0$, it follows from the discussion in $\S 1.1 .3$ that the fibers of $\phi$ are geodesics (since they have vanishing mean curvature vector). Moreover, now the identity $\omega_{i 0}=$ $-r_{0} \omega_{i}$ holds, so it follows that $d \omega_{0}=0$, i.e., the leaves of $\omega_{0}=0$ are the frame bundles of hypersurfaces in $M$ that are orthogonal to the fibers of $\phi$. By the structure equations, the second fundamental form $H$ of each such leaf is the restriction to that leaf of the tensor

$$
\mathbf{H}=\omega_{i 0} \circ \omega_{i}=-r_{0} \omega_{i} \circ \omega_{i}=-r_{0} g^{\prime}
$$

where $g^{\prime}$ is the usual orthogonal projection of the metric $g$. Since $\mathbf{H}$ is a scalar multiple of $g^{\prime}$, the induced metric on these orthogonal hypersurface leaves, it follows that each such hypersurface is totally umbilic.

Suppose now that the second possibility holds. Since $r_{0}=0$, it follows that $\rho=\rho^{\prime}=r_{i} \omega_{i}$ is a closed 1-form that is well-defined on $M$. Since $M$ is connected and simply connected, there exists a smooth positive function $r$ on $M$, unique up to a constant scalar multiple, so that $r^{-1} d r=\rho$. Set $\mathbf{X}=r^{n-2} \mathbf{u}$. Now a calculation using the equation $\omega_{i 0}=-(n-2) r_{i} \omega_{0}+a_{i j} \omega_{j}$ yields

$$
\mathcal{L}_{\mathbf{X}} g=0 .
$$

Thus $\mathbf{X}$ is a Killing vector field for $g$, as desired.

Remark 1. Remark The two possibilities are not quite mutually exclusive. There is essentially one local example that falls under both Types. This is when $r_{0}=r_{i}=a_{i j}=0$. By the structure equation $d r_{0}=\left(r_{0}^{2}+K\right) \omega_{0}$, this can only happen when $K=0$. Then the foliation of $\phi$-fibers is by parallel geodesics and the orthogonal hypersurfaces are totally geodesic. In this case, the morphism $\phi$ is locally equivalent to the standard linear orthogonal projection $\phi: \mathbb{R}^{n+1} \rightarrow \mathbb{R}^{n}$.

\subsection{Examples and further results.}

Theorem 3 forms the basis of a classification of the harmonic morphisms of corank 1 for which the domain has constant sectional curvature.

3.3.1. Umbilic morphisms. Because of the nature of the orthogonal foliation, I will say that a harmonic morphism $\phi:\left(M^{n+1}, g\right) \rightarrow\left(N^{n}, h\right)$ whose corresponding integral manifold is of Type 1 is an umbilic morphism. Thus, in this case, the fibers of $\phi$ are the geodesics orthogonal to a foliation of $M$ 
by parallel totally umbilic hypersurfaces. To specify the map $\phi$ up to obvious equivalences, it is evidently enough to specify a single totally umbilic hypersurface in $M$. The number of possible cases depends on the sign of the sectional curvature $K$. For simplicity, I will only treat the cases $K=1,0,-1$.

If $K=1$ and $M=S^{n+1}$ with its standard metric, then every totally umbilic hypersurface is an $n$-sphere of radius at most 1 . The family of parallel hypersurfaces consist of the parallel hyperspheres, one of which is a great $n$-sphere. Using the standard inclusions of $S^{p}$ into $\mathbb{R}^{n+1}$, the formula for the map $\phi$ becomes

$$
\phi\left(x_{0}, \ldots, x_{n+1}\right)=\frac{\left(x_{0}, \ldots, x_{n}\right)}{\sqrt{1-x_{n+1}^{2}}},
$$

which is undefined at the 'poles', $x_{n+1}= \pm 1$.

If $K=0$ and $M=\mathbb{R}^{n+1}$, there are two types of umbilic foliations. The first type is by parallel planes, leading to the harmonic morphism

$$
\phi\left(x_{0}, \ldots, x_{n+1}\right)=\left(x_{0}, \ldots, x_{n}\right),
$$

and the second is the radial projection $\phi: \mathbb{R}^{n+1} \backslash \mathbf{0} \rightarrow S^{n}$ given by

$$
\phi\left(x_{1}, \ldots, x_{n+1}\right)=\frac{\left(x_{1}, \ldots, x_{n}\right)}{\sqrt{x_{1}^{2}+\cdots+x_{n+1}^{2}}} .
$$

If $K=-1$, and $M=D^{n+1} \subset \mathbb{R}^{n+1}$, the hyperbolic ball, then there are three types of umbilic foliations, leading to three types of umbilic morphisms. The first type is when one of the umbilic hypersurfaces is totally geodesic. Then the parallel hypersurfaces are all totally umbilic with principal curvatures less than 1 in absolute value. The parallel mapping identifying any two such hypersurfaces is a homothety and the quotient metric is of constant negative sectional curvature. Thus, the corresponding $\phi$ is a harmonic morphism from $D^{n+1}$ to $D^{n}$. The second type is when all of the parallel umbilic hypersurfaces have principal curvature equal to +1 or -1 . Of course, in this case, the hypersurfaces are all horocycles tangent at a unique common point on the ideal boundary of $D^{n+1}$. The metric on such a horocycle is the flat metric, so this gives rise to a harmonic morphism $\phi: D^{n+1} \rightarrow \mathbb{R}^{n}$. (This example is more simply seen in coordinates as the linear projection from the upper half space model of hyperbolic space to the boundary plane.) Finally, the third type is when all of the parallel umbilic hypersurfaces have principal curvatures of absolute value greater than 1 . In this case, the hypersurfaces are the level sets of the distance function from 
some fixed point $z \in D^{n+1}$ and hence are isometric to standard spheres $S^{n}$ of varying radii. The corresponding harmonic morphism is well-defined as a $\operatorname{map} \phi: D \backslash\{z\} \rightarrow S^{n}$.

By passing to quotients and covers and so forth, every example with $M$ complete or compact can be constructed from these examples. Note that by taking appropriate quotients by discrete subgroups, one can construct compact examples only by starting with the harmonic morphisms from $\mathbb{R}^{n+1}$ or $D^{n+1}$ to $\mathbb{R}^{n}$ since these are the only ones for which $r_{0}$ can satisfy the equation $d r_{0}=\left(r_{0}^{2}+K\right) \omega_{0}$ on a compact fiber of $\phi$.

All of these examples of umbilic morphisms are due to Gudmundsson, who also proved a characterization theorem [Gu1,Theorem 3.6] asserting that a non-constant harmonic morphism $\phi:\left(M^{n+1}, g\right) \rightarrow(N, h)$ where both $g$ and $h$ have constant sectional curvature that has the additional property that the conformal factor $r$ is constant on curves in $M$ perpendicular to the fibers of $\phi$ must be one of these examples or else $r$ is actually constant.

3.3.2. Isometric quotient morphisms. Finally, consider the case where there exists a non-zero Killing field $\mathbf{X}$ on $M$ whose integral curves are the fibers of the harmonic morphism $\phi$. From the discussion of this example in $\S 2.1$, the metric $h$ on the target manifold $N$ must satisfy $\phi^{*}(h)=$ $c|\mathbf{X}|^{2 /(n-2)} g^{\prime}$ for some constant $c>0$, where, as usual, $g^{\prime}$ denotes the part of the metric $g$ that is orthogonal to the fibers of $\phi$. If $M$ is oriented and $*_{g} 1$ denotes the volume form of $g$, then a computation shows that the pulled-back volume form for $h$ must have the form

$$
\left.\phi^{*}\left(*_{h} 1\right)=c^{n / 2}|\mathbf{X}|^{2 /(n-2)}(\mathbf{X}\lrcorner *_{g} 1\right) .
$$

Note that the $n$-form on the right hand side of this equation is smooth away from the zero locus $Z \subset M$ of $\mathbf{X}$.

Because $\mathbf{X}$ is a non-zero Killing field, $Z$ must be a smooth, proper submanifold of even codimension, say, $2 q \leq n+1$. In fact, in a neighborhood of any $z \in Z$, there exists a geodesic normal coordinate system $y_{1}, \ldots, y_{n+1}$ on a $z$-neighborhood $U$ so that $Z \cap U$ is defined by the equations $y_{1}=\cdots=y_{2 q}=0$ and $\mathbf{X}$ has the form

$$
\mathbf{X}=m_{1}\left(y_{1} \frac{\partial}{\partial y_{2}}-y_{2} \frac{\partial}{\partial y_{1}}\right)+\cdots+m_{q}\left(y_{2 q-1} \frac{\partial}{\partial y_{2 q}}-y_{2 q} \frac{\partial}{\partial y_{2 q-1}}\right)
$$

where $m_{i}>0$. (To derive this normal form, note that since the flow of $\mathbf{X}$ is an isometry fixing $z \in Z$, it induces a one-parameter group of rotations on $T_{z} M$. This one-parameter subgroup of $\mathrm{O}\left(T_{z} M\right)$ is then generated by 
a linear vector field $\mathbf{X}_{z}$ on $T_{z} M$. Taking the usual normal form of such a linear vector field with respect to an orthogonal basis of $T_{z} M$ and then using the fact that the exponential map $\exp _{z}: T_{z} M \rightarrow M$, which is a local diffeomorphism on a neighborhood of $z$, must carry $\mathbf{X}_{z}$ to $\mathbf{X}$ then yields the above normal form. Note that, by this constuction, the submanifold $Z$ near $z$ is the image under $\exp _{z}$ of a linear subspace of $T_{z} M$ of even codimension.)

It follows that the expression $\left.|\mathrm{X}|^{2 /(n-2)}(\mathrm{X}\lrcorner *_{g} 1\right)$ cannot be smooth on a neighborhood of $z$ unless $n=3$. Of course, when $n=3$, this expression actually is smooth, a fact that will be used below. Moreover, when $n=3$, the formula for the 'push-down' metric simplifies to

$$
\phi^{*}(h)=c\left(|\mathbf{X}|^{2} g-\left(\mathbf{X}^{b}\right)^{2}\right)
$$

where $\mathbf{X}^{\mathrm{b}}$ represents the $g$-dual 1-form to $\mathbf{X}$.

When $M^{n+1}$ is a simply connected manifold and $g$ is complete with constant sectional curvature $K$, there are many Killing vector fields, each leading to a different harmonic quotient. Of course, these quotients will usually have singularities, since the space of integral curves of a general Killing vector field on $M$ will not, in general, carry the structure of a smooth manifold. Thus, it will usually be necessary to restrict to an open set $U \subset M$ on which the integral curves of $\mathbf{X}$ form an amenable foliation before one can construct the target manifold.

Example 2. Spherical quotients. Consider the case $K=1$, so that $M=$ $S^{n+1} \subset \mathbb{R}^{n+2}$ (embedded as the standard unit sphere $x_{0}{ }^{2}+\cdots+x_{n+1}{ }^{2}=1$ ). Then, in order for the integral curves of $\mathbf{X}$ to be closed (so that the space of integral curves endowed with the quotient topology is at least Hausdorff), it must be conjugate in the rotation group to a multiple of a vector field of the form

$$
\mathbf{X}=m_{0}\left(x_{0} \frac{\partial}{\partial x_{1}}-x_{1} \frac{\partial}{\partial x_{0}}\right)+\cdots+m_{k}\left(x_{2 k} \frac{\partial}{\partial x_{2 k+1}}-x_{2 k+1} \frac{\partial}{\partial x_{2 k}}\right)
$$

for some integer $k$ satisfying $2 k \leq n$ and some positive integers $m_{0} \leq \cdots \leq$ $m_{k}$ with greatest common divisor equal to 1 . Take $\mathbf{X}$ to have this form.

The generic integral curve of $\mathbf{X}$ is of period $2 \pi$ and there will be ramified integral curves (that are not fixed points) unless $m_{i}=1$ for all $i$. The fixed point set $Z \subset S^{n+1}$ will be empty unless $2 k<n$, in which case $Z=S^{n-2 k-1}$ is defined by the equations $x_{0}=x_{1}=\cdots=x_{2 k+1}=0$. Note that, away from $Z$, the $\mathbf{X}$-orthogonal part $g^{\prime}$ of the induced metric $g$ is smooth, as is the tensor $|\mathrm{X}|^{2 /(n-2)} g^{\prime}$. 
If $R \subset S^{n+1}$ denotes the closed subset of $S^{n+1}$ consisting of the ramified integral curves of $\mathbf{X}$ plus the fixed points, then setting $N=\left(S^{n+1} \backslash R\right) / \mathbf{X}$, with quotient projection $\phi: S^{n+1} \backslash R \rightarrow N$, defines a smooth $n$-manifold on which there exists a unique Riemannian metric $h$ that satisfies $\phi^{*}(h)=$ $|\mathbf{X}|^{2 /(n-2)} g^{\prime}$. With respect to this metric, $\phi:\left(S^{n+1} \backslash Z, g\right) \rightarrow(N, h)$ is a harmonic morphism.

Note that since $|\mathbf{X}|^{2 /(n-2)} g^{\prime}$ does not vanish on $R \backslash Z$, it is not possible to extend $N$ as a smooth manifold in such a way that $\phi$ can be extended over any part of $R \backslash Z$ as a smooth mapping. Thus, if $\phi$ is to extend globally to $S^{n+1}$ as a harmonic morphism to a smooth $n$-manifold, then $R=Z$, i.e., $m_{i}=1$ for all $i$. Assume this from now on, so that $R=Z$.

Now, if $Z$ is empty, i.e., if $n=2 k$, then $\mathbf{X}$ generates the standard circle action on $S^{2 k+1}$ and the quotient manifold is $N=\mathbb{C P}^{k}$, with the FubiniStudy metric.

On the other hand, if $Z=S^{n-2 k-1}$ is non-empty, it consists of either 1 or 2 components. Since the expression $\left.|\mathbf{X}|^{2 /(n-2)}(\mathbf{X}\lrcorner *_{g} 1\right)$ is not smooth along $Z$ unless $n=3$, it follows that there are at most two cases to consider: The cases $(n, k)=(3,0)$ and $(n, k)=(3,1)$. In the first case, $N$ can be completed to a topological manifold $\bar{N}$ by adding a single point corresponding to the fixed point set $Z=S^{2}$ and, in the second case, $N$ can be completed to a topological manifold $\bar{N}$ by adding two points, corresponding to the two points of $Z=S^{0}$. However, in neither case can the smooth structure on $N$ be extended across the extra point(s) so that the metric $h$ extends as a smooth metric on $\bar{N}$. In fact, in each case, the metric on $N$ is invariant under an action of $\mathrm{SO}(3)$, so that in a neighborhood of one of the extra points, 'polar coordinates' can be introduced so that $s$ represents the distance from the singular point and a neighborhood of the extra point can be written in the form $(0, \epsilon) \times S^{2}$ with the metric taking the form

$$
d s^{2}+f(s) \sigma
$$

where $\sigma$ is the standard metric of curvature 1 on $S^{2}$. In the case that $k=0$, the function $f$ has the form $f(s)=s(s+1)^{2}(s+2)$ near $s=0$ (the extra point) while in the case that $k=1$, the function $f$ has the form $f(s)=$ $s^{2}(1-s / 2)^{2}$ near $s=0$. In neither case is $f$ the square of a smooth, odd function of $s$ whose derivative at $s=0$ equals 1 , a necessary and sufficient condition for such a metric to represent a smooth, rotationally invariant metric in polar coordinates.

The conclusion of all this discussion is that the only non-constant harmonic morphism whose domain is the entire $S^{n+1}$ with its standard 
metric and whose range is a smooth $n$-manifold is the standard fibering $\phi: S^{2 k+1} \rightarrow \mathbb{C P}^{k}$. Note that this analysis gives a more comprehensive solution to the problem discussed in [Do].

Remark: Other examples. Using the above discussion as a guide, it is possible to write down an $\mathrm{SO}(4)$-invariant metric $g$ on $S^{4} \subset \mathbb{R}^{5}$ that admits a Killing field $\mathbf{X}$ with two isolated fixed points so that the quotient space $N$ carries the structure of a smooth manifold diffeomorphic to $S^{3}$ endowed with a smooth metric $h$, so that the leaf projection $\phi:\left(S^{4}, g\right) \rightarrow\left(S^{3}, h\right)$ is a harmonic morphism with exactly two singular points. However, the metric $g$ cannot have constant sectional curvature.

\section{Example 3. Euclidean quotients.}

The non-zero Killing vector fields on $\mathbb{R}^{n+1}$ can be divided into two types, those without fixed points and those with fixed points.

If $\mathbf{X}$ is a Killing field on $\mathbb{R}^{n+1}$ without fixed points, then, up to a constant multiple, it it conjugate by an Euclidean motion to the vector field

$$
\mathbf{X}=\frac{\partial}{\partial x_{0}}+m_{1}\left(x_{1} \frac{\partial}{\partial x_{2}}-x_{2} \frac{\partial}{\partial x_{1}}\right)+\cdots+m_{q}\left(x_{2 q-1} \frac{\partial}{\partial x_{2 q}}-x_{2 q} \frac{\partial}{\partial x_{2 q-1}}\right)
$$

for some positive real numbers $m_{1}, \ldots, m_{q}$ where $q \leq n / 2$. From now on, assume that $\mathbf{X}$ has this form. Note that all of the integral curves of $\mathbf{X}$ intersect the hyperplane $x_{0}=0$ exactly once, and do so transversely, so that this hyperplane can be taken as the model space for the quotient manifold $N$. It is not difficult to see that the metric $h$ on $N$ (canonically determined up to a constant scalar factor) that makes the leaf projection $\phi: \mathbb{R}^{n+1} \rightarrow N=\mathbb{R}^{n}$ a harmonic morphism is never of constant sectional curvature unless $q=0$, in which case $\phi$ is simply the linear orthogonal projection $\phi: \mathbb{R}^{n+1} \rightarrow \mathbb{R}^{n}$.

If $\mathbf{X}$ is a Killing field on $\mathbb{R}^{n+1}$ with fixed points, then, it is conjugate by an Euclidean motion to the vector field

$$
\mathbf{X}=m_{1}\left(x_{1} \frac{\partial}{\partial x_{2}}-x_{2} \frac{\partial}{\partial x_{1}}\right)+\cdots+m_{q}\left(x_{2 q-1} \frac{\partial}{\partial x_{2 q}}-x_{2 q} \frac{\partial}{\partial x_{2 q-1}}\right)
$$

for some positive real numbers $m_{1}, \ldots, m_{q}$ where $q \leq(n+1) / 2$. (For simplicity, I am taking $x_{1}, \ldots, x_{n+1}$ as an orthogonal linear set of coordinates on $\mathbb{R}^{n+1}$.)

By the same analysis as in the previous example, the quotient space $N$ cannot be defined as a Hausdorff space unless the ratios of the $m_{i}$ are rational, in which case, they can be taken to be integers with greatest common 
divisor equal to 1 . Then, the ramification set $R$ will be larger than the zero set $Z$ unless $m_{i}=1$ for all $i$ and it will not be possible to define a smooth structure on any extension of $N$ so that the leaf quotient $\phi: \mathbb{R}^{n+1} \backslash R \rightarrow N$ extends as a smooth map to any part of $R \backslash Z$. Thus, if the harmonic morphism is to be globally defined, one must have $R=Z$, i.e., all of the $m_{i}=1$.

Now, as has already been noted, the map $\phi$ cannot be extended smoothly across $Z$ unless $n=3$, so that either $q=1$ or $q=2$. With some effort, it can be shown that the first case leads to a singular quotient that cannot be smoothed. However, for $q=2$, the Killing field

$$
\mathbf{X}=x_{1} \frac{\partial}{\partial x_{2}}-x_{2} \frac{\partial}{\partial x_{1}}+x_{3} \frac{\partial}{\partial x_{4}}-x_{4} \frac{\partial}{\partial x_{3}}
$$

does lead to a smooth quotient, for setting

$$
\begin{aligned}
& y_{1}=\frac{1}{2}\left(x_{1}^{2}+x_{2}^{2}-x_{3}^{2}-x_{4}^{2}\right) \\
& y_{2}=x_{1} x_{4}-x_{2} x_{3} \\
& y_{3}=x_{1} x_{3}+x_{2} x_{4}
\end{aligned}
$$

yields a map $\phi=\left(y_{1}, y_{2}, y_{3}\right): \mathbb{R}^{4} \rightarrow \mathbb{R}^{3}$ that satisfies

$$
\phi^{*}(h)=|\mathbf{X}|^{2} g-\left(\mathbf{X}^{b}\right)^{2}
$$

where $g$ and $h$ are the standard metrics on $\mathbb{R}^{4}$ and $\mathbb{R}^{3}$, respectively. Thus, this defines a smooth harmonic morphism, globally defined on $\mathbb{R}^{4}$. Note that this is an example of a harmonic morphism defined by quadratic maps, as studied in [OW]. In [Ba], it was shown that this is essentially the only harmonic map from $\mathbb{R}^{4}$ to $\mathbb{R}^{3}$ given by quadratic polynomials.

\section{Example 4. Hyperbolic quotients.}

The discussion in this last example will be more cursory than in the previous examples since the results are very like the first two and proved by the same methods.

Again, just as in the Euclidean case, if $\left(M^{n+1}, g\right)$ is the hyperbolic ball, there are two kinds of non-zero Killing vector fields. The first kind have no fixed points, while the second have a fixed submanifold which is totally geodesic.

If $\mathbf{X}$ is a Killing field on $M$ without fixed points, then, just as in the Euclidean case, one can choose a hypersurface $N$ in $M$ that is transverse to the vector field $\mathbf{X}$ and so that each integral curve of $X$ in $M$ meets $N$ in 
exactly one point. This $N$ is diffeomorphic to $\mathbb{R}^{n}$, of course, and there will be a unique induced metric on $N$ so that the leaf projection is a harmonic morphism.

If $\mathrm{X}$ is a Killing field on $M$ that has a non-empty fixed point set $Z$, then, again, unless $n=3$, the harmonic morphism defined on $X \backslash Z$ cannot be extended smoothly across the fixed point set. However, even when $n=3$, the two cases that arise do not yield a smooth quotient, being more like the two cases that arose in the study of $S^{4}$ than the two cases that arose in the study of $\mathbb{R}^{4}$. Thus, none of these give rise to global harmonic morphisms on hyperbolic 4-space.

\section{References.}

[Ba] P. Baird, Harmonic morphisms and circle actions on 3-and 4-manifolds, Ann. Inst. Fourier (Grenoble) 40 (1990), 177-212.

[BW1] P. Baird and J. C. Wood, Harmonic morphisms and conformal foliations by geodesics of three-dimensional space forms, J. Austral. Math. Soc. 51 (1991), 118-153.

[BW2] P. Baird and J. C. Wood, The geometry of a pair of Riemannian foliations by geodesics and associated harmonic morphisms, Bull. Soc. Math. Belg., Ser. B 44 (1992), 115-139.

[BCG] R. Bryant, et al., Exterior Differential Systems, Springer-Verlag, New York. 1991.

[Do] Y. X. Dong, A Bernstein theorem for harmonic morphisms from a $N+1$-sphere to a $N$-manifold, preprint, Hangzhou University 1996.

[EL1] J. Eells and L. Lemaire, A report on harmonic maps, Bull. London Math. Soc. 10 (1978), 1-68.

[EL2] J. Eells and L. Lemaire, Another report on harmonic maps, Bull. London. Math. Soc. 20 (1988), 385-524.

[Fu] B. Fuglede, Harmonic morphisms between Riemannian manifolds, Ann. Inst. Fourier 28 (1978), 107-144.

[Gu1] S. Gudmundsson, Harmonic morphisms between spaces of constant curvature, Proc. Edinb. Math. Soc. 36 (1992), 133-143.

[Gu2] §. Gudmundsson, On the existence of harmonic morphisms from symmetric spaces of rank one preprint, University of Lund 1996. 
[Is] T. Ishihara, A mapping of Riemannian manifolds which preserves harmonic functions, J. Math. Kyoto Univ. 19 (1979), 215-229.

[OW] Y. L. Ou and J. C. Wood, On the classification of quadratic harmonic morphisms between Euclidean spaces, Algebras, Groups and Geometries 13 (1996), 41-53.

[Sp] M. Spivak, A Comprehensive Introduction to Differential Geometry, Publish or Perish, Inc. Wilmington, Del. 1979. (For a discussion of the moving frame, especially see Volume III, Chapters 1 and 2.)

[W1] J. C. Wood, On the Gauss map of a harmonic morphism, Research Notes in Mathematics 131, Pitman, London 1985, 149-155.

[W2] J. C. Wood, Harmonic morphisms between Riemannian manifolds, in Geometry and Global Analysis, Eds. T. Kotake, S. Nishikawa and R. Schoen, Tohoku Univ. Sendai 1993, 413-422.

DUKE UNIVERSITY,

PO Box 90320,

DURHAM, NC 27708-0320

E-mail address: bryant@math.duke.edu 